\title{
SEISMIC RISK SENSITIVITY OF STRUCTURES EQUIPPED WITH ANTI-SEISMIC DEVICES WITH UNCERTAIN PROPERTIES
}

\author{
Fabrizio Scozzese ${ }^{\text {a, }}$, , Andrea Dall'Asta $^{\text {a }}$, Enrico Tubaldi ${ }^{b}$ \\ ${ }^{\text {a }}$ School of Architecture and Design, University of Camerino, Viale della Rimembranza, 63100 Ascoli Piceno (AP), Italy. \\ ${ }^{\mathrm{b}}$ University of Strathclyde, 16 Richmond St., G1 1XQ Glasgow, United Kingdom. \\ E-mail addresses: fabrizioscozzese@gmail.com, andrea.dallasta@unicam.it, etubaldi@gmail.com
}

\begin{abstract}
Damping and isolation devices are often employed to control and enhance the seismic performance of structural systems. However, the effectiveness of these devices in mitigating the seismic risk may be significantly affected by manufacturing tolerances, and systems equipped with devices whose properties deviate from the nominal ones may exhibit a performance very different than expected.

The paper analyzes this problem by proposing a general framework for investigating the sensitivity of the seismic risk of structural systems with respect to system properties varying in a prescribed range. The proposed framework is based on the solution of a reliability-based optimization (RBO) problem, aimed to search for the worst combination of the uncertain anti-seismic device parameters, within the allowed range of variation, that maximizes the seismic demand hazard. A hybrid probabilistic approach is employed to speed up the reliability analyses required for evaluating the objective function at each iteration of the RBO process. This approach combines a conditional method for estimating the seismic demand at a given intensity level, with a simulation approach for representing the seismic hazard.

The proposed method is applied to evaluate the influence of the variability of the properties of linear and nonlinear fluid viscous dampers on the seismic risk of a low-rise steel building. The study results show that the various response parameters considered are differently affected by the damper property and unveil the capability of the proposed approach to evaluate the potentially worst conditions that jeopardize the system reliability.
\end{abstract}

\section{KEY WORDS: Reliability-based optimization; Seismic risk sensitivity; IM-based approach; Viscous dampers; Subset Simulation; Structural engineering.}

\section{Introduction}

The evaluation of the seismic reliability of a structural system requires the characterization of the uncertainty in the seismic input as well as in the structure geometrical and mechanical properties, and the propagation of these uncertainties to assess the structural failure probability, typically expressed as the probability of exceeding specified levels of the monitored response parameters [1,2]. While the seismic input uncertainty generally significantly influences the seismic risk [3-6], the effect of the structural properties must be evaluated on a case-by-case basis [3,7-9]. Isolated structures or structures equipped with dampers fall into this category of structures whose performance may be significantly affected by uncertainties other than the seismic one, because their seismic response depends mainly on the characteristics of a few number of devices. Furthermore, seismic isolation and energy dissipation devices have properties which can vary significantly as a function of manufacturing process, time, temperature, load history, strain-rate and velocity, among others [10], thus differing from the nominal ones considered for the design. Seismic design codes (ASCE/SEI-7; ASCE/SEI 41-13; EN 15129) [11-13] aim at controlling this issue by two actions: a) providing some acceptance criteria, limiting the deviation of the device behavior with respect to the nominal one, b) introducing property modification factors in the design. In particular, the property modification factors are employed to modify the device properties in the system response assessment [14] and their values are calibrated considering both the permitted tolerance range and the safety levels to be achieved. Differently from the case of traditional structural materials like concrete or masonry, in the case of seismic devices such as isolators or dampers, statistical information about mechanical properties are not available. This is due to several reasons: the device properties notably change batch by batch [15-17], the dispersion within each batch can significantly vary with the manufacturer, the number of devices produced in a single batch is often too limited to allow a statistical analysis. These issues motivate a safety check based on a different approach, aiming at seeking the worst conditions within the property range allowed by the tolerance, and foster studies on risk assessment methodologies that do not strictly comply with a probability distribution [18,19]. Local and Global sensitivity analysis could also be used to study the influence of the variability of these system parameters on the performance of a model $[20,21]$. These methods generally do not require assigning a probabilistic distribution of the model parameters, but they necessitate

${ }^{1}$ Corresponding author at: Department of Civil and Building Engineering and Architecture, Polytechnic University of Marche, Via Brecce Bianche Ancona (AN), Italy 
the specification of the domain of parameter variations. For example, the FOSM method [22,23] can be used to evaluate the seismic demand sensitivity around a reference configuration. This method has been employed in Lee and Mosalam (2005) [5] to evaluate the sensitivity of the seismic response of a reinforced concrete shear-wall building with respect to the model and seismic input parameters, and in Lupoi et. al (2006) [24] to evaluate the seismic fragility of structural systems by also including model parameter uncertainty. Au (2005) [25] proposed a method to obtain statistical information about the sensitivity of the reliability of structures with respect to different ranges of model parameters, based on the solution of an augmented reliability problem, in which the system parameters are modelled as random variables with fictitious probability distribution, and on the use of Subset Simulation [26]. Such strategy can be useful for a preliminary sensitivity investigation, as done for instance in the work of Dall'Asta et al. (2016) [27] to explore the influence of viscous dampers with variable properties on the seismic response of simple structural systems represented by a single degree of freedom (SDOF) model. However, further insights are needed to establish this influence on more realistic structural systems.

Studies on the sensitivity of the performance of systems with respect to isolator and damper properties are very limited. Zona et al. (2012) [28] carried out a sensitivity-based study of the influence of brace over-strength distributions on the seismic response of steel frames with buckling-restrained braces (BRBs). However, their analysis focuses only on the mean response under a set of seismic records with a given intensity, thus disregarding the uncertainty of the response due to record-to-record variability effects. Jensen et al. $(2009,2011)$ [29,30] and Taflanidis and Beck (2009) [31] proposed two reliability-based design methodologies for the optimal sizing of passive energy dissipation systems for the seismic protection of structures. These methodologies are based on the evaluation of the sensitivity of the reliability of the system with respect to the damper design parameters, an information that can be used to understand how damper parameter variations in the neighborhood of the design values affect the risk estimate.

This paper proposes an original approach for exploring the sensitivity of the seismic risk of structural systems with respect to uncertain system parameters, by formulating this problem as an optimization one. In particular, the evaluation of the combination of the worst-case scenario of uncertain parameter values that maximize the risk variation is cast in the form of a constrained reliability-based optimization (RBO) problem, which can be efficiently solved by employing efficient and already available optimization techniques (e.g., [32,33]). It is worth to point out that the term "sensitivity" is used here in a general way, as it does not describe the local relationship between model parameter variations and output variations [20,21], but the relationship between the admissible set of parameter variations and the consequent maximum output variation.

Another element of novelty in this work is represented by the probabilistic approach proposed for estimating the seismic risk within the RBO framework, combining Subset Simulation [26] with multiple-stripe analysis (MSA) [34]. This approach allows reducing the number of simulations required to solve the reliability problem with respect to standard SS, without affecting the accuracy of the results.

In order to evaluate the capability of the proposed RBO framework to identify the critical combinations of parameter variations, a structural system, often adopted as a benchmark in the literature to evaluate the efficiency of seismic response control devices [35$38]$, is considered. The structure is upgraded by using a widely diffused dissipative system, consisting of viscous devices with linear or nonlinear behavior, whose behavior is described by the combination of two constitutive parameters $(c$ and $\alpha)$. These devices may have properties different from the nominal ones considered at the design stage, because of the manufacturing process. To cope with such uncertainty, the main international codes [11-13] provide some acceptance criteria according to which fluid viscous devices are tested to ensure that their responses, generally expressed in terms of force-velocity relation, do not deviate from the nominal design condition more than a tolerance. However, no prescriptions or limits are imposed on the viscous constitutive parameters, whose admissible (i.e., complying with the tolerance provided on the force response) ranges of variability are unknown. Very few studies have been carried out thus far on this topic [2,39], and the applications proposed in this work contribute to fill the gap in the technical literature on the effects of the uncertainty of nonlinear fluid viscous damper properties on the seismic performance of structures.

The paper is organized as follows. First, the probabilistic framework is introduced by furnishing details about the formulation of the RBO problem, the probabilistic tools used for the performance assessment and the optimization solver. Subsequently, the specific problem of the risk sensitivity analysis of structural systems equipped with fluid viscous dampers is introduced by considering both the cases of linear dampers and nonlinear dampers. The benchmark case study is then discussed by describing the model, the seismic scenario, and the dampers design criteria. The last two sections illustrate and discuss the solution of two RBO problems: the former assumes that the damper variability concerns only the viscous coefficients, and the latter assumes that the damper variability concerns also the response coefficients $\alpha$ controlling the damper nonlinear behaviour. In the last part of the paper, some conclusions are drawn, commenting on the proposed methodology and the specific problem analysed.

\section{Methodology}

\subsection{Seismic performance assessment of structural systems with uncertain input and model properties}

The seismic design and assessment of structures aims at ensuring that the probability of having an unsatisfactory performance (often referred to as failure) is lower than a reference acceptable level. This level is prefixed by seismic codes and can be tailored to the 
type of structure at hand, its function, and the consequences of failure [11,40,41]. With reference to ordinary civil structures, different limit states and levels of the allowed exceedance probability are introduced to control the performance. Conventional thresholds are specified for these limit states, and the mean annual frequencies (MAFs) of exceedance approximately vary from $1 \cdot 10^{-2} \mathrm{yrs}^{-1}$ for serviceability limit states to $1-2 \cdot 10^{-3} \mathrm{yrs}^{-1}$ for ultimate limit states $[11,40,41]$, while safety checks against collapse should be oriented to ensure a mean annual failure rate lower than $10^{-5}-10^{-6}[11,28]$.

In general, the seismic structural performance is evaluated by monitoring a set of engineering demand parameters (EDPs) relevant to the system at hand, and the seismic checks aim to verify that the mean annual frequency (MAF) $v_{D}\left(d_{f}\right)$ of exceeding a prefixed threshold value $d_{f}$ is lower than an acceptable limit $v_{0}$ (depending on the particular limit state or performance condition, as discussed above). Design codes [11,40,41] and practical assessment procedures follow an "intensity-based assessment approach" [42], aimed at satisfying the aforementioned reliability condition in an indirect and simplified way, avoiding probabilistic analyses. For this purpose, a conventional seismic response measure $d^{*}$ is evaluated, via structural analysis, under a seismic input with assigned intensity, and then it is verified that $d^{*}<d_{f}$. Different threshold values of $d_{f}$, each associated to a performance objective, are coupled with the various intensity levels.

A more rigorous performance assessment should consider explicitly the seismic demand hazard function, $v_{D}(d)$, expressing the MAF of exceeding different values $d$ of the global and local EDP relevant to the performance of the analyzed system [42]. Obviously, in the evaluation of $v_{D}(d)$, all the sources of uncertainty involved in the problem shall be accounted for. In this regard, it may be convenient to consider two separate vectors for describing these uncertainties: $\boldsymbol{X} \in \boldsymbol{\Omega}$ is the vector collecting the random variables representing the ground motion and the structural system uncertainties, which can be described by assigning a probability density function, and $\boldsymbol{\theta}=\left[\theta_{1}, \ldots, \theta_{n}\right] \in \Gamma$ is the vector of all the other parameters affecting the system performance, but for which a probabilistic model is not available. These parameters, assumed independent from $\boldsymbol{X}$, are hereinafter referred to as design variables (DVs), and the corresponding nominal values are denoted as $\boldsymbol{\theta}_{0}$.

In this study, a sensitivity study is carried out to evaluate explicitly how $\boldsymbol{\theta}$ affects the seismic demand hazard. In particular, if $\boldsymbol{x}$ denotes the realization of $\boldsymbol{X}$, and $d(\boldsymbol{x} \mid \boldsymbol{\theta})$ denotes the generic demand, conditional to a given combination of model parameters $\boldsymbol{\theta}$, the corresponding demand hazard function $v_{D}(d \mid \boldsymbol{\theta})$ can be estimated through the following reliability integral

$$
v_{D}(d \mid \boldsymbol{\theta})=v_{\min } \int_{\Omega} I_{d}(\boldsymbol{x} \mid \boldsymbol{\theta}) p_{\boldsymbol{X}}(\boldsymbol{x}) d x
$$

where $p_{X}(\boldsymbol{x})$ is the joint probability density function (PDF) of $\boldsymbol{X}$, and $I_{d}(\boldsymbol{x} \mid \boldsymbol{\theta})$ is an indicator function, such that $I_{d}=1$ if $d(\boldsymbol{x} \mid \boldsymbol{\theta})>$ $d^{*}$, otherwise $I_{d}=0$. The multiplicative term $v_{\min }$ is the MAF of occurrence of a seismic event of any significant magnitude [33]. A simple way to investigate the influence of $\boldsymbol{\theta}$ on the system seismic demand hazard could be that of performing several reliability analyses, i.e., solving the problem of Eq. (1) for different sets of $\boldsymbol{\theta}$ values. Monte Carlo techniques [26,43,44] can be employed for solving the integral for each combination of the parameters, but this type of approach is generally computationally expensive, and it becomes unfeasible in the problem considered in the application, where the possible combinations could be numerous.

\subsection{Constrained optimization problem}

In this study, the search for the maximum effects of design parameter variations on the generic seismic demand hazard $v_{D}(d \mid \boldsymbol{\theta})$ is cast in the form of a constrained reliability-based optimization (RBO) problem. In particular, the objective function $f_{O b j}$ that must be maximized is expressed as the variation of the MAF of exceedance of an EDP value $d^{*}$, normalized with respect to the MAF of exceedance corresponding to the reference design values $\boldsymbol{\theta}_{0}$ :

$$
f_{O b j}\left(\boldsymbol{\theta}, d^{*}\right)=\frac{v_{D}\left(d^{*} \mid \boldsymbol{\theta}\right)-v_{D}\left(d^{*} \mid \boldsymbol{\theta}_{0}\right)}{v_{D}\left(d^{*} \mid \boldsymbol{\theta}_{0}\right)}
$$

in which $v_{D}\left(d^{*} \mid \boldsymbol{\theta}\right)$ and $v_{D}\left(d^{*} \mid \boldsymbol{\theta}_{0}\right)$ denote the MAFs of exceedance corresponding, respectively, to the varied and the nominal DVs (Fig. 1).

A constraint is used to control the range of variation of the DVs, by assigning lower and upper bounds to $\boldsymbol{\theta}$, or more in general via constraint functions $g_{j}(\theta)(j=1, \ldots, k)$.

The constrained RBO problem can be formulated as follows,

$$
\left\{\begin{array}{l}
\max _{\boldsymbol{\theta}} \quad f_{O b j}\left(\boldsymbol{\theta}, d^{*}\right)=\frac{v_{D}\left(d^{*} \mid \boldsymbol{\theta}\right)-v_{D}\left(d^{*} \mid \boldsymbol{\theta}_{0}\right)}{v_{D}\left(d^{*} \mid \boldsymbol{\theta}_{0}\right)} \\
g_{j}(\boldsymbol{\theta}) \leq 0 \quad(j=1, \ldots, k)
\end{array}\right.
$$

which allows to find the "optimal" combination of DVs leading to the highest increments of the MAF of exceedance for the reference monitored performance measure. 
It is noteworthy that in general the maximum variations of the various EDPs of interest are obtained for different combinations of the DVs. Thus, a single RBO problem need to be solved for each of the monitored response variables.

a)

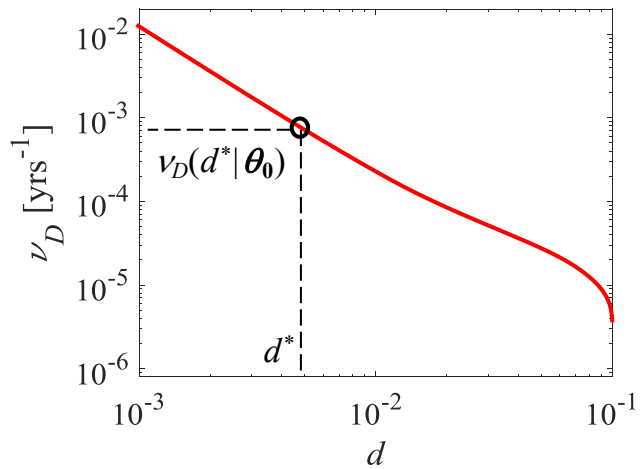

b)

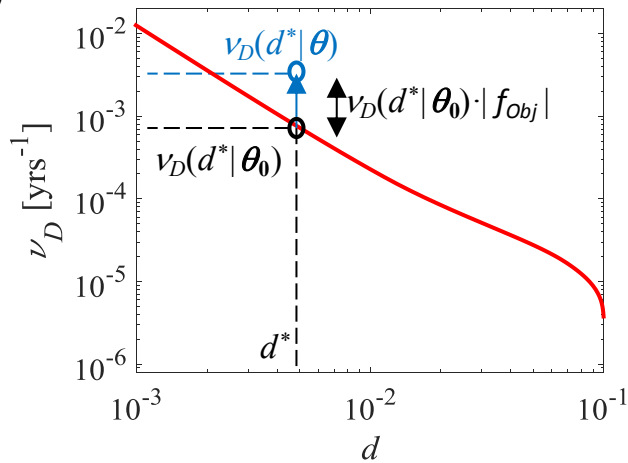

Fig. 1. a) MAF of exceedance of the EDP corresponding to the nominal values of the DVs; b) increment of the risk of exceeding the threshold value $d^{*}$ due to the allowed variability on the model parameters.

\subsection{Hybrid reliability approach for seismic demand estimation}

The RBO problem of Eqn. (3) is solved by employing a nested double-loop approach [33], consisting of an outer loop where optimization is carried out, and an inner loop which is used at each iteration to evaluate $v_{D}\left(d^{*} \mid \boldsymbol{\theta}\right)$. This solution approach is computationally very expensive, particularly because the inner loop must be invoked many times before converging to the optimal solution. Thus, an efficient probabilistic method is employed here to achieve accurate estimates of $v_{D}\left(d^{*} \mid \boldsymbol{\theta}\right)$ while limiting the number of simulations. Such method, denoted hereinafter as hybrid, is based on a conditional probabilistic approach [42] for evaluating the seismic demand at different seismic intensity levels, and on the use of Subset Simulation for defining the seismic hazard. More precisely, a stochastic ground motion model is considered, and Subset Simulation is employed [26]), to derive the IM hazard curve, $v_{I M}(\mathrm{im})$, up to very small rates of exceedances. For each intensity level, a set of ground motion samples is selected, and these samples are then used to evaluate the response statistics and build a seismic demand model conditional to the seismic intensity level via multiple-stripe analysis (MSA) [34]. This demand model, represented by $G_{D \mid I M}(d \mid \boldsymbol{\theta}$, im), expresses the probability of exceeding the demand value $d$, conditional to $\boldsymbol{\theta}$ and to the seismic intensity level $\mathrm{im}$, and can be estimated via a parametric method, i.e., by assuming the widely employed [45,46] lognormal distribution for describing the response $d$ for each value of $\boldsymbol{\theta}$ and each IM level.

The hazard function and the conditional demand can be convolved together, by exploiting the Total Probability Theorem, to evaluate the mean annual rate of exceedance $v_{D}\left(d^{*} \mid \boldsymbol{\theta}\right)[42]$ :

$$
v_{D}\left(d^{*} \mid \boldsymbol{\theta}\right)=\int_{I M} G_{D \mid I M}\left(d^{*} \mid \boldsymbol{\theta}, i m\right)\left|d v_{I M}\right|
$$

The integral of Eq. (4) is computed numerically, by employing a trapezoidal rule.

It is noteworthy that the idea of combining Subset Simulation with a conditional probabilistic approach has the advantage of reducing the variance of the estimation of the performance compared to a direct approach based on SS. Moreover, Subset Simulation automatically performs the hazard disaggregation at different IM levels, providing a set of stochastic ground motion samples conditional to different non-overlapping IM intervals. Hence, there is no need of scaling the records [47], and the seismological features of the earthquake samples change consistently with the seismic intensity level. A similar hybrid approach has been recently used by Bradley et al. 2015 [48] for testing different ground motion selection methods. However, differently from that work, employing a pure Monte Carlo approach for IM hazard curve construction, in this study the more efficient Subset Simulation [26] is used to reach lower annual rates of exceedance and thus simulate ground motion samples conditional to rarer seismic intensities. The efficiency of Subset Simulation may be further improved by employing recently developed algorithms [ref1, ref2] for generating the candidate states. However, in the present study the original Markov Chain Monte Carlo algorithm and the Metropolis-Hastings sampler proposed in [26] have been used.

\subsection{Optimization solver}

The COBYLA (Constrained Optimization By Linear Approximation) gradient-free optimization algorithm, developed by Powell (2007) [32] and implemented in OpenCossan [49], is used to solve the constrained optimization problems. COBYLA exploits a sequential trust-region algorithm based on linear approximations on both the objective function and the constraint functions: the approximations consist of linear interpolation at $n+1$ points (the vertices of the trust-region), where $n$ represents the dimension of 
the space of (design) variables. Although the rate of convergence of COBYLA is slower than that of gradient-based algorithms, its robustness and the low number of parameters that need to be tuned for performing optimization make this algorithm suitable for the purposes of the present applications.

The size of the trust region is controlled by the algorithm and it is decreases towards convergence. The initial and final values of the trust region's radius are problem-dependent, and in this work they are set equal to $0.20 \cdot \boldsymbol{\theta}$ and $10^{-6} \cdot \boldsymbol{\theta}$, respectively. Further details on COBYLA algorithm and on the values of the parameters can be found in Powell (2007) [32], in the OpenCossan documentation [49], as well as in Altieri et al. (2017) [33].

\section{Fluid viscous dampers with variable properties}

Fluid viscous dampers are dissipation devices widely used for seismic response control, due to their effectiveness in increasing the damping capacity of a structural system, hence notably reducing its response in terms of both inter-story drifts and absolute accelerations $[50,51]$. The force-velocity constitutive law of these devices can be described through the following relationship [5254]:

$$
F_{d}(v)=c|v|^{\alpha} \operatorname{sgn}(v)
$$

where $v$ is the velocity between the device's ends, $F_{d}$ is the damper resisting force, $|v|$ is the absolute value of $v$, sgn is the sign operator, $c$ and $\alpha$ are two constitutive parameters: the former is a multiplicative factor, while the latter describes the damper nonlinear behaviour.

The main international seismic codes [11-13] acknowledge that the manufacturing process is characterized by some uncertainty affecting the viscous constitutive parameters, whose actual values might differ from the nominal ones used in the design. To cope with such uncertainty, some acceptance criteria are provided. In particular, the ASCE/SEI 41-13 [12] and the European code EN 15129 [13] require that the maximum experimental force $F_{d}(v)$ exhibited by the damper tested under harmonic displacement timehistories, deviates from the expected (design) value, $F_{d}{ }^{*}(v)$, by no more than a tolerance $p$ within a range of velocities $v$ spanning from zero to the maximum design one $v^{*}$. This requirement can be formulated in terms of the following inequality:

$$
(1-p) F_{d}^{*}(v) \leq F_{d}(v) \leq(1+p) F_{d}^{*}(v), \quad v \leq v \leq v^{*}
$$

where $p=15 \%$ according to the abovementioned seismic standards. The safety check should be coherently carried out by employing a lower/upper bound approach, considering the worst conditions compatible with the acceptance criteria.

It is worth to note that, as explicitly declared in ASCE/SEI 41-13 [12] (Section C14.3.2.4, Upper-and Lower-Bound Design and Analysis Properties) and implicitly assumed by EN 15129 [13] (Section E.2, Design Requirements), the effects of dampers' uncertainty are only considered on the viscous coefficient $c$, while the exponent $\alpha$ is assumed as fixed. However, such assumption (variability on $c$ only) is not justified in either of these two seismic codes, and furthermore, in ASCE/SEI 41-13 [12] (Section C14.3.2.4) it is explicitly written that: "The authors recognize that much of the data needed to rationally develop the required individual factors for energy dissipation devices do not exist at the time of writing".

In light of the aforementioned gap in the regulatory framework, and given the scarce literature available on this topic [2,39], the application proposed in this paper aims at investigating the influence of the variability of the viscous damper properties, including that of $\alpha$, on the seismic risk. The application proposed below can be viewed as an extension of the work carried out in Dall'Asta et al. (2017) [2] to the case of real structures equipped with multiple devices.

The RBO problem presented in Section 2.2 (Eq. (3)) is defined below for the specific case in which the DVs are represented by the viscous constitutive properties of $n$ fluid viscous dampers, i.e., $\boldsymbol{c}=\left[c_{1}, \ldots, c_{n}\right]$ and $\alpha=\left[\alpha_{1}, \ldots, \alpha_{n}\right]$. In particular, two different types of constrained reliability-based optimization problems are considered, as described below in detail.

\subsection{RBO problem 1}

In RBO problem 1, the velocity exponents $\boldsymbol{\alpha}$ are kept fixed and equal to the nominal values $\boldsymbol{\alpha}_{0}$, and only the viscous coefficients $\boldsymbol{c}$ are assumed as design variables. These coefficients are allowed to deviate from the nominal values $\boldsymbol{c}_{0}$ while satisfying the inequality constraint of Eq. (6) on the damper forces. This results in a range of variation $\left[-0.85 \boldsymbol{c}_{0}, 1.15 \boldsymbol{c}_{0}\right]$ for $\boldsymbol{c}$. In fact, variations of $\boldsymbol{c}$ provide a homogeneous effect on the damper response for the whole range of velocities, with normalized force variations equal to the normalized viscous coefficient variations.

The constrained RBO problem can be formulated as follows,

$$
\left\{\begin{array}{c}
\max \quad f_{O b j}\left(\boldsymbol{c}, d^{*}\right)=\frac{v_{D}\left(d^{*} \mid \boldsymbol{c}\right)-v_{D}\left(d^{*} \mid \boldsymbol{c}_{0}\right)}{v_{D}\left(d^{*} \mid \boldsymbol{c}_{0}\right)} \\
-0.85 c_{0, i} \leq c_{i} \leq 1.15 c_{0, i}(i=1, \ldots, n)
\end{array}\right.
$$

in which the objective function, $f_{O b j}$, is expressed as the normalized variation of the MAF of exceedance of some reference values of the monitored EDP, $d^{*}$. 
The problem solution allows finding the "optimal" combination of the varied viscous coefficients $\boldsymbol{c}$ that maximizes the objective function, i.e., that corresponds to the highest increment of the MAF of exceeding the reference value of the monitored performance parameter. On this regard, five EDPs are assumed to characterize the system's seismic performance, namely the maximum interstory drift, related to the structural damage; the maximum absolute base-shear of the frame only (dampers contribution excluded); the maximum absolute acceleration, related to damage of acceleration-sensitive nonstructural components; the maximum force and stroke in the dampers, related to the cost, the size, and the reliability of the viscous dampers $[33,55]$. Thus, the RBO problem of Eq. (7) has to be solved five times.

\subsection{RBO problem 2}

In RBO problem 2, both the damper constitutive parameters are allowed to vary while satisfying the acceptance criterion of Eq. (6). In this problem, differently from RBO problem 1 , the link between the dampers force variations and the perturbed viscous parameters is not straightforward, and joint variations $(\widehat{\boldsymbol{\alpha}}, \widehat{\boldsymbol{c}})$ of the constitutive parameters, such that $\boldsymbol{\alpha}=\boldsymbol{\alpha}_{0}+\widehat{\boldsymbol{\alpha}}$ and $\boldsymbol{c}=\boldsymbol{c}_{0}+\widehat{\boldsymbol{c}}$, must be considered. Among the various combinations compatible with the constraints, one will yield the maximum variation of the demand hazard.

Fig. 2 shows the normalized force-velocity relations corresponding to a linear damper (a) and a nonlinear damper (b), obtained for the design nominal parameters $\left(\alpha_{0}, c_{0}\right)$ (black solid line). On the same figure, the upper and lower bounds of the allowed response variability are also illustrated (red solid lines), corresponding to the case with viscous coefficient variations $\hat{c}=+/-15 \%$ and $\hat{\alpha}=$ 0 . Moreover, the varied response curves obtained for two specific pairs of admissible perturbed parameters combinations $(\hat{\alpha}, \hat{c})$ are superimposed: the dashed blue curve represents the maximum admissible positive variation of the exponent $\alpha$, corresponding to the condition in which the normalized response variation attains the upper bound value (i.e., $F_{d} / F_{d} *=1.15$ ) at the normalized design velocity (i.e., $v / v^{*}=1$ ); the dotted blue curve represents the maximum admissible negative variation of the exponent $\alpha$, corresponding to the condition in which the response variation attains the lower bound value (i.e., $F_{d} / F_{d} *=0.85$ ) at the design velocity (i.e., $\left.v / v^{*}=1\right)$. Such combined variations of the viscous properties are both complying with the tolerances for velocity values lower than the design one $\left(v / v^{*}=1\right.$ in the normalized axis), as required by the code [12]. However, for velocity values beyond the design one (i.e., $v / v^{*}>1$ ), the perturbed force assumes values outside the upper/lower bounds (red solid lines), and the specific trend depends on the sign of $\hat{\alpha}$, which governs the rate of change of the nonlinear response, with non-homogeneous effects along the range of velocity.

a)

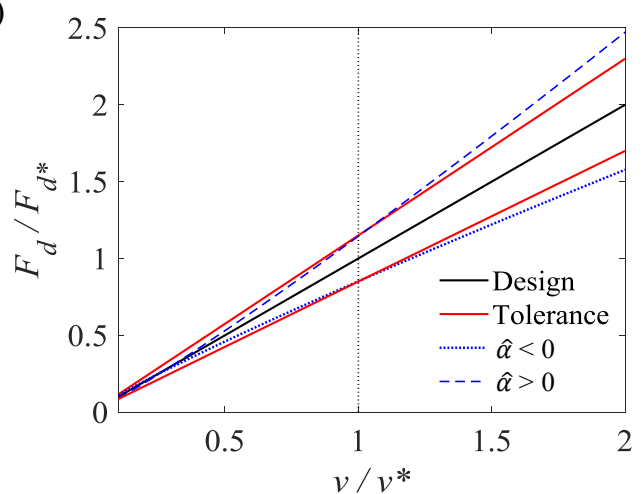

b)

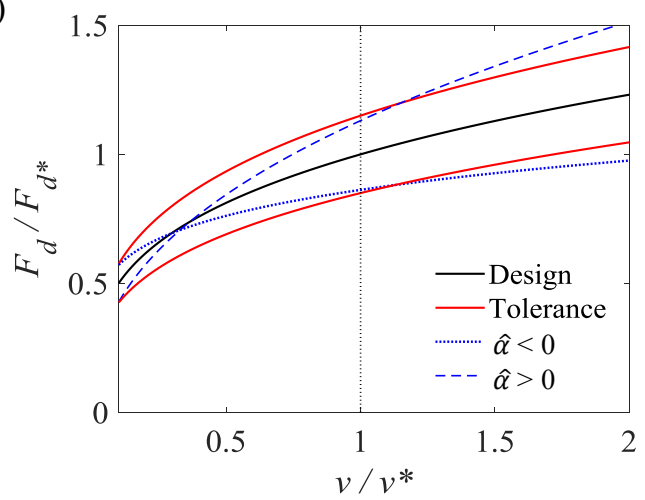

Fig. 2. Effect of code-complying damper parameters variability in terms of device force response: a) linear damper with $\alpha_{0}=1.0$, and b) nonlinear damper with $\alpha_{0}=0.3$.

For the sake of completeness, the effect of variable viscous parameters on the dissipated energy is also presented. The expression of the energy dissipated in a sinusoidal cycle with circular frequency $\omega$ and amplitude $v / \omega$ is as follows,

$$
W_{d}=\frac{c v^{\alpha+1}}{\omega} \lambda(\alpha)
$$

where $\lambda(\alpha)$ is a geometric function related to the shape of the cycle $[54,56]$.

Fig. 3 reports the relationships between $W_{d}$, normalized by the energy dissipated at the design velocity value $v^{*}$ (by a system with the nominal parameters $c_{0}$ and $\alpha_{0}$ ), and the velocity $v$, for all the cases discussed above. These are the nominal design conditions (black solid line), the admissible perturbed parameters combinations with $\hat{\alpha}<0$ (blue dotted line) and $\hat{\alpha}>0$ (blue dashed line), and the upper and lower bound conditions (red solid lines), corresponding to the case with $\hat{c}=+/-0.15 c_{0}$ and $\hat{\alpha}=0$. As already observed for the force response, the effects of combined variations of $c$ and $\alpha$ might lead to unexpected values of energy dissipation, i.e., the case with $\hat{\alpha}<0$ might reduce the dissipative capacity at the higher velocities with respect to the case with nominal parameters, while the case with $\hat{\alpha}>0$ might lead to higher damper forces (in the range $v / v^{*}>1$ ). 
The trends observed in Fig. 2 and Fig. 3 allow making some qualitative and preliminary observations on the response of viscous devices for seismic actions other than the design one. In fact, if $v / v^{*}=1$ represents the reference seismic design condition, it is possible to observe that the expected damper response (in terms of both force and dissipated energy) exhibits variations of different amplitude and signs, depending on whether the seismic intensity, and thus the velocity, is higher or lower than the design one.

a)

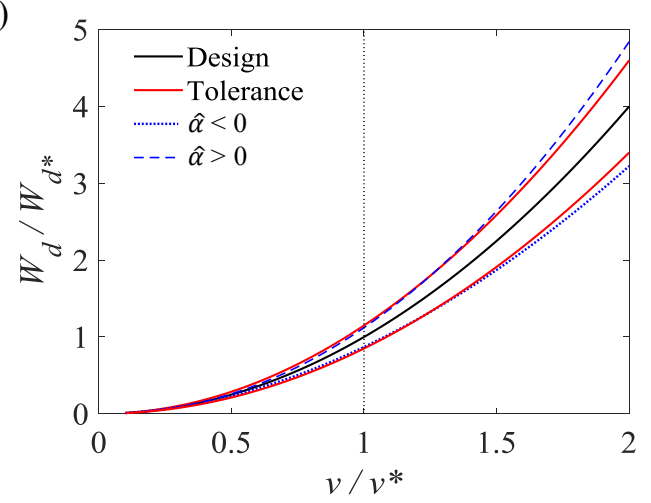

b)

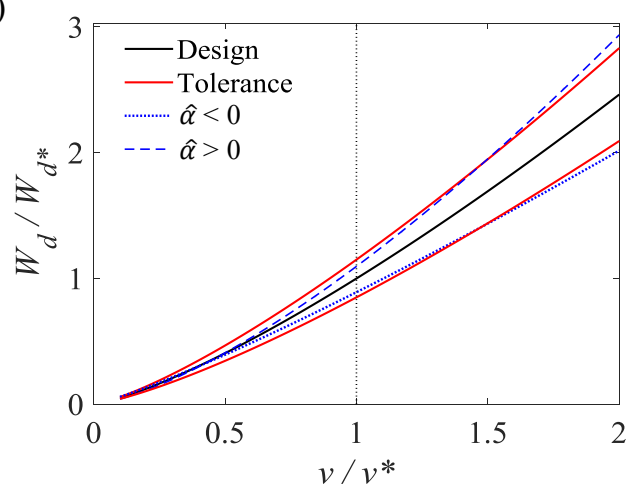

Fig. 3. Effect of code-complying damper parameters variability in terms of energy dissipation (per cycle): a) linear damper with $\alpha_{0}=1.0$, and b) nonlinear damper with $\alpha_{0}=0.3$.

In RBO problem 2, the design parameters are both $\mathbf{c}$ and $\boldsymbol{\alpha}$, and their domain of variation are subject to the constraints defined above.

The constrained RBO problem is formulated as follows,

$$
\left\{\begin{array}{l}
\max \quad f_{o b j}\left(\boldsymbol{c}, \boldsymbol{\alpha}, d^{*}\right)=\left[\frac{v_{D}\left(d^{*} \mid \boldsymbol{c}, \boldsymbol{\alpha}\right)-v_{D}\left(d^{*} \mid \boldsymbol{c}_{\mathbf{0}}, \boldsymbol{\alpha}_{\mathbf{0}}\right)}{v_{D}\left(d^{*} \mid \boldsymbol{c}_{\mathbf{0}}, \boldsymbol{\alpha}_{\mathbf{0}}\right)}\right] \\
\mid\left(c_{0, i}+\hat{c}_{i}\right) v^{\left(\alpha_{0, i}+\widehat{\alpha}_{i}\right)}-c_{0, i} v^{\alpha_{0, i} \mid} \leq 0.15 c_{0, i} v^{\alpha_{0, i}} \quad \forall 0 \leq v \leq v^{*} \quad(i=1, \ldots, n)
\end{array}\right.
$$

in which the constraint is expressed in terms of damper forces within the range of velocity from 0 to $v^{*}$ (maximum design velocity). The objective function, $f_{O b j}$, similarly to the previous case, expresses the normalized variation of the MAF of exceedance of a reference value of the monitored EDP, $d^{*}$.

\section{Benchmark case study and dampers design}

In this section, the benchmark structural system and seismic scenario employed for illustrating the application of the proposed framework for sensitivity analysis are presented first. Subsequently, two retrofit interventions involving either linear or nonlinear viscous dampers are considered. The criteria followed for designing the dampers are presented and the nominal design properties ( $\boldsymbol{c}_{\mathbf{0}}$ and $\boldsymbol{\alpha}_{\mathbf{0}}$ ) of the linear and nonlinear devices are furnished. Then, the probabilistic response at the reference conditions (i.e., when the dampers have nominal viscous properties) is illustrated, showing the demand hazard curves for the various EDPs of interest and the values of the MAF of exceedance corresponding to the design performance levels $v_{D}\left(d^{*} \mid \boldsymbol{c}_{\mathbf{0}}, \boldsymbol{\alpha}_{\mathbf{0}}\right)$.

\subsection{Case study description}

The case study consists of a 3-storey steel moment-resisting frame building, designed within the SAC Phase II Steel Project, and widely used as benchmark structure in several other works concerning structural response control [35-38]. The structural system was designed for gravity, wind, and seismic loads in order to conform to local code requirements in Los Angeles, California region. As shown in Fig. 4, the whole structural system consists of perimeter moment-resisting frames and internal gravity frames with shear connections, while the structural model for analysis purposes is a two-dimensional frame representing one half of the structure in the north-south direction. The main geometrical details and the size of the steel members (wide-flange sections are used for both columns and beams) are shown in Fig. 4. Further details concerning the structural geometry and loads can be found in [36].

a)

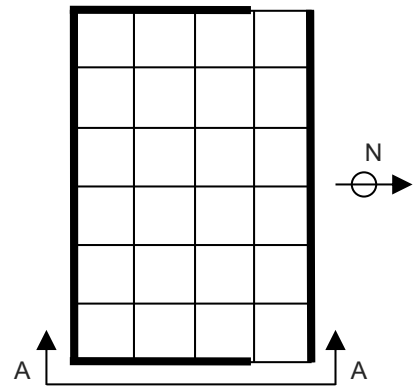

b)

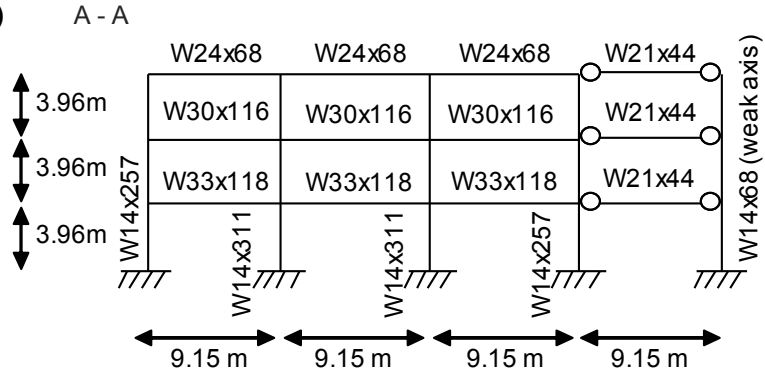

Fig. 4. a) Plan (thick lines highlight moment-resisting frames) and b) elevation of the 3-storey steel frame from the SAC Phase II Steel Project. 
The finite element model of the system is developed in OpenSees [57] following the approach described in [35] and briefly recalled below. A distributed plasticity approach is adopted [58-60], with nonlinear force-based elements and fiber sections with Steel02 uniaxial material. An elastic fictitious P-delta column (not shown in Fig. 4) is introduced to account for the nonlinear geometrical effects induced by the relevant vertical loads, those carried by the inner (not explicitly modelled) gravity frames included. A corotational approach for the system's coordinate transformation is used to perform large displacement (small strain) analysis. The strength and deformability of panel zones are neglected. The elastic damping properties are accounted for through the Rayleigh model by assigning a $2 \%$ damping ratio at the first and second vibration modes. The estimated vibration periods $T_{i}$, reported in Table 1.

Table 1. Vibration periods for the 3-storey steel moment-resisting frame.

\begin{tabular}{ll}
\hline Mode & $T_{i}[\mathrm{~s}]$ \\
\hline 1 & 0.995 \\
2 & 0.325 \\
3 & 0.173 \\
\hline
\end{tabular}

\subsection{Seismic scenario}

Being the structure designed for the Los Angeles area, the Atkinson-Silva (2000) [61] source-based ground motion model for California region is used to characterize the seismic hazard at the building's site. This model, combined with the stochastic point source simulation method of Boore (2003) [62], is considered for generating ground motion samples. The seismic scenario is described by two seismological parameters, the moment magnitude $M$, and the source-to-site (epicentral) distance $r$, which are modelled as random variables. The magnitude is characterized by the Gutenberg-Richter probability density function $f_{M}(m)$

$$
f_{M}(m)=\beta \frac{e^{-\beta\left(m-m_{0}\right)}}{1-e^{-\beta\left(m_{\max }-m_{0}\right)}}
$$

bounded within the interval $\left[m_{0}, m_{\max }\right]=[5,8]$ and with parameter $\beta=2.303$; the epicentral distance is modelled according to the $\operatorname{PDF} f_{R}(r)$

$$
f_{R}(r)=\left\{\begin{array}{cr}
\frac{2 r}{r_{\max }} \text { if } & 0 \leq r<r_{\max } \\
0 & r \geq r_{\max }
\end{array}\right.
$$

under the hypothesis that the source produces random earthquakes with equal likelihood anywhere within a distance from the site $r_{\max }=50 \mathrm{~km}$, beyond which the seismic effects are assumed to become negligible. The soil condition is described by a deterministic value of the shear-wave velocity parameter $V_{S 30}=310 \mathrm{~m} / \mathrm{s}$, representative of average soil condition in the considered area [52].

Fig. 5 illustrates the ground motion total radiation (Fourier) spectra $A(\omega)$, and the time-envelope functions $e(t)$, obtained for different earthquake moment magnitudes $M(5,6.5,8)$ and fixed epicentral distance $r=20 \mathrm{~km}$. Fig. 6 shows the effect of the epicentral distance $r(2,30,50 \mathrm{~km})$, for a fixed magnitude $M=6$. It is worth to note that the present seismic scenario is also consistent with several other works $[26,63]$ in which the Atkinson-Silva ground motion model is adopted, and further details about the parameters used in the present study can be found in Dall'Asta et al. (2017) [2].

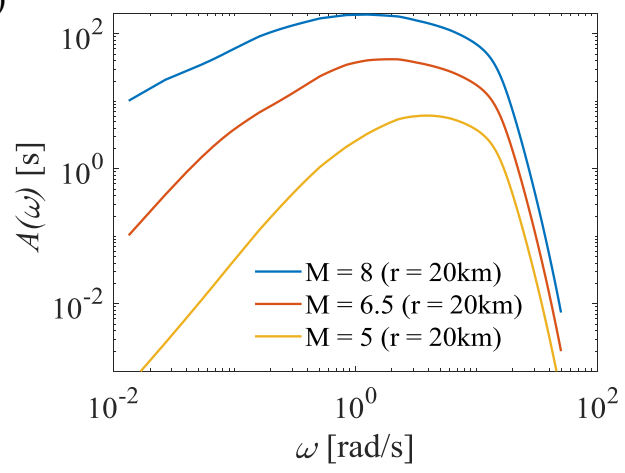

b)

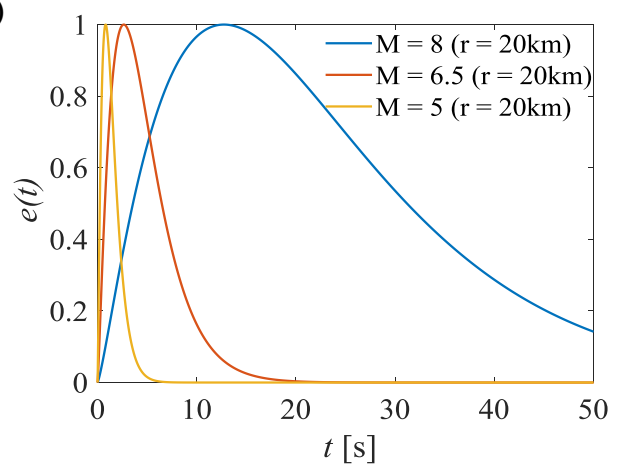

Fig. 5. a) Total radiation Fourier spectra and b) time-envelope functions for $r=20 \mathrm{~km}$ and different earthquake moment magnitudes. 
a)

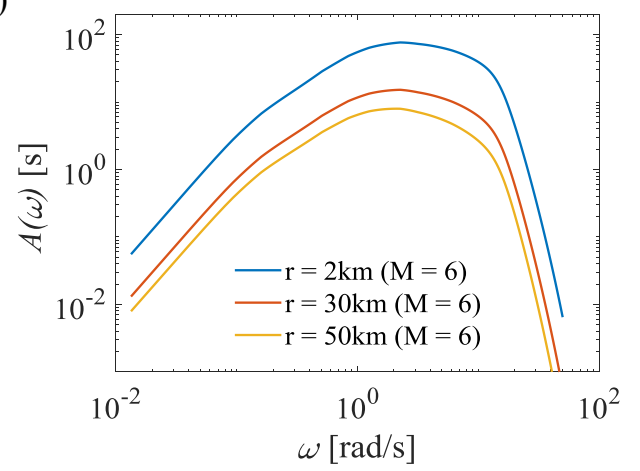

b)

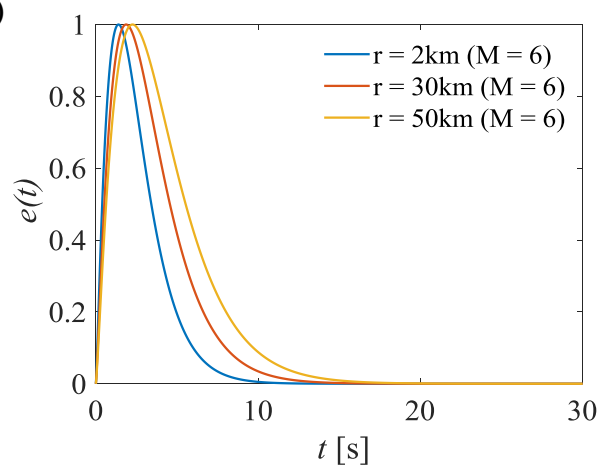

Fig. 6. a) Total radiation Fourier spectra and b) time-envelope functions for $M=6$ and different epicentral distances.

Besides the scenario-related random variables (magnitude and distance), further uncertainties derive from the record-to-record variability expected for ground motions associated with seismic inputs with the same magnitude and distance. This variability is described first by generating the signals as realizations of a white-noise process, and then by including an additional source of variability through the multiplicative factor of the radiation spectra, $\varepsilon_{m o d}$, proposed by Jalayer and Beck (2008) [63]. This factor is assumed to be lognormally-distributed, with a lognormal mean equal to 0 and a lognormal standard deviation of 0.5.

The pseudo-spectral acceleration $S_{a}\left(T_{1}\right)$ at the fundamental period $T_{1}=1.0 \mathrm{~s}$ is assumed as IM, and the corresponding hazard curve is obtained by a single-run of Subset Simulation, carried out by considering 20 simulation levels, each having a target intermediate exceedance probability $p_{0}=0.5$, and $N=500$ samples per level. Fig. 7 shows the hazard curve, with the 21 IM interval boundaries indicated by black vertical lines.

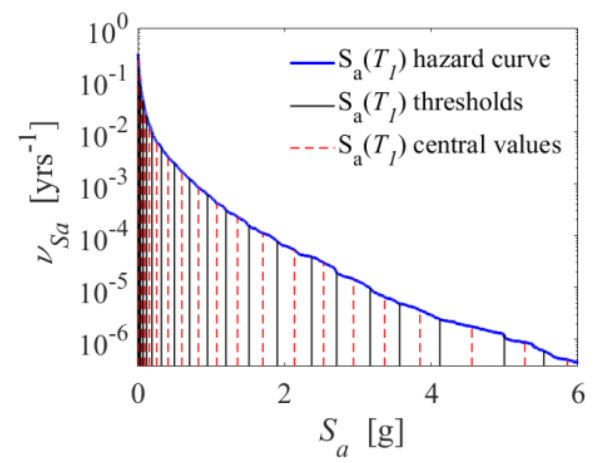

Fig. 7. Hazard curve for the IM $S_{a}\left(T_{1}\right)$ obtained via Subset Simulation.

\subsection{Performance criteria and dampers design}

The dampers are designed to achieve an enhanced building performance level according to ASCE/SEI 41-13 [12], consisting of meeting the immediate occupancy requirements (performance level 1-B) at the BSE-2E seismic hazard level (i.e., with probability of exceedance equal to $5 \%$ in 50 years, corresponding to the annual rate of exceeding $v_{0}=0.001$ ).

The dampers are placed into the structural frame (Fig. 8) connected in series with steel supporting braces, and two different cases are studied: linear viscous dampers $\left(\alpha_{0}=1.0\right)$ and nonlinear viscous dampers $\left(\alpha_{0}=0.3\right)$. For the purpose of the dampers' design, the structural performance is described in terms of a global EDP, represented by the interstory drift ratio, whose limit value at the Immediate Occupancy Limit State is assumed equal to 0.01 as suggested in FEMA-350 [64] (Table 4-10 of the aforementioned code) for low-rise ordinary moment-resisting steel buildings.

Following a deterministic design approach, commonly employed in practice, the dampers' viscous coefficients $c_{0 \mathrm{i}}(i=1,2,3$ floor levels) are calibrated to reduce the mean value of the maximum interstory drift demand ( $\mu_{\text {drift max }}$ ), evaluated for a set of 7 accelerograms whose intensity, defined in terms of $\mathrm{IM}=S_{a}\left(T_{1}\right)$, is consistent with the reference hazard level (i.e., with $v_{0}=0.001$ $\left.\mathrm{yrs}^{-1}\right)$. The IM value corresponding to the exceedance frequency considered for the design is equal to $\operatorname{IM}\left(v_{0}\right)=S_{a}\left(T_{1}\right)=0.77 \mathrm{~g}$. The subset of 7 simulated ground motion time-histories is selected from the set of samples stored during execution of Subset Simulation, and the selection criterion is such that it satisfies (without scaling) the spectrum compatibility at the building's first period $T_{1}=1.0 \mathrm{~s}$. The target performance, achieved for both the linear and nonlinear dampers case, is equal to $\mu_{\text {drift } \max }=0.0097 \mathrm{rad}<0.01 \mathrm{rad}$, corresponding to a $40 \%$ reduction with respect to the bare frame performance. The supporting braces are designed to withstand (without buckling) the damper force amplified according to the following expression [12],

$$
F_{b}=F_{d}\left(2 \mu_{v e l, \text { max }}\right)=c_{0}\left|2 \mu_{v e l, \text { max }}\right|^{\alpha}
$$


corresponding to the force provided by the damper at a velocity value twice the mean maximum velocity $\mu_{v e l, m a x}$ observed under the set of 7 ground motions representing the design scenario at the MAF of exceedance $v_{0}$.

There are several methods available $[65,66]$ for distributing the dampers at the various storeys of the building. Some methods assume predefined damper distributions, e.g., the viscous coefficients of the dampers are uniformly distributed or distributed proportionally to the storey stiffness or shear. Being the differences observed among standard methods usually not very large [66], the shearproportional distribution is assumed in this study, for its simplicity and because it has been shown to provide satisfactory results [67]. To be precise, a distribution of $c_{0, i}$ at the different storeys proportional to the shear distribution (i.e., from the top below, respectively, $0.54,0.86,1.00)$ of the first mode of vibration is assumed. The nominal properties of the viscous coefficients are reported in Table 2 (with labels related to Fig. 8) together with the axial stiffness values of the steel braces.

Assuming a S275 steel grade cold-formed profiles, the following hollow squared cross-sections are employed for the supporting braces: $350 \times 16 \mathrm{~mm}$ (where $350 \mathrm{~mm}$ denotes the external dimensions and $16 \mathrm{~mm}$ the thickness) for the braces connected to linear dampers, and 300x10 $\mathrm{mm}$ for those connected to the nonlinear ones. It can be observed that the use of nonlinear dampers allows to reduce the braces' sections compared to the case of linear dampers.

In the finite element analyses, a Maxwell element consisting of an elastic spring (with stiffness $k_{b}$ ) and a viscous dashpot in series is used to describe the behaviour of the damper-brace systems.

It is noteworthy that the design of the damper properties could have been carried out by considering performance-based criteria rather than a deterministic criterion, and by employing advanced optimization procedures $[33,50,67,68]$. Indeed, the same tools used here for the sensitivity analysis could be employed for the damper design.

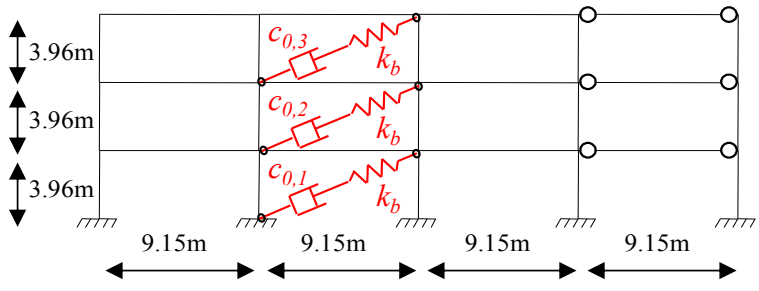

Fig. 8. Maxwell model for the brace-damper systems: viscous dampers are represented as dashpots with coefficients $c_{0, \mathrm{i}}$ while the supporting braces as elastic springs with finite stiffness $k$ b.

Table 2. Damper parameters and brace stiffness with linear and nonlinear dampers.

\begin{tabular}{lll}
\hline Parameters & $\alpha_{0}=1.00$ & $\alpha_{0}=0.30$ \\
\hline$c_{0,1}\left[\mathrm{kN} \cdot \mathrm{s}^{\alpha} / \mathrm{m}^{\alpha}\right]$ & 8500 & 2350 \\
$c_{0,2}\left[\mathrm{kN} \cdot \mathrm{s}^{\alpha} / \mathrm{m}^{\alpha}\right]$ & 7310 & 2021 \\
$c_{0,3}\left[\mathrm{kN} \cdot \mathrm{s}^{\alpha} / \mathrm{m}^{\alpha}\right]$ & 4590 & 1269 \\
$k_{\mathrm{b}}[\mathrm{kN} / \mathrm{mm}]$ & 450.19 & 244.30 \\
\hline
\end{tabular}

The performance of the structure is evaluated by monitoring the following global EDPs: the maximum interstory drift among the various storeys, $I D R$, the maximum absolute base-shear carried out by the frame only, $V_{b}$, and the maximum absolute acceleration among the various floors, $A$. The dampers performance is monitored by considering the following two local EDPs, accounting for the cost, the size and the failure of the devices: the maximum absolute force of the dampers placed at the first storey, $F_{d l}$, which carries the largest forces; and the maximum stroke, $\Delta_{d l}$, of the damper at the first storey. It is worth to note that the maximum interstory drift is kinematically related to the maximum stroke among the various floors, whereas the maximum damper velocities are related to damper forces through Eq. (5).

Table 3 collects the design values $d^{*}$ of the five monitored EDPs, all obtained under the design seismic scenario except for the stroke and force of the dampers, whose design values are further amplified according to the rules provided in ASCE/SEI 41-13 [12]: the design forces are computed via Eq. (12), and coherently the strokes are assumed as twice the values obtained under the design seismic scenario. The design values of the maximum dampers' velocity, necessary to define the tolerance range described by Eq. (6), are $v^{*}=0.27 \mathrm{~m} / \mathrm{s}$ and $v^{*}=0.30 \mathrm{~m} / \mathrm{s}$ for, respectively, linear and nonlinear dampers.

\subsection{Reference seismic demand: dampers with nominal properties}

The performance of the systems corresponding to linear and nonlinear dampers with nominal properties $\left(\alpha_{0, i}, c_{0, i}\right)$ is evaluated by performing MSA in accordance with Section 2.3. The intensity levels at which MSA is performed are indicated by red dashed lines in Fig. 7, and represent the central values of the IM intervals considered for SS. Among the $N=500$ ground motion time-series generated at each simulation level, a subset made of the 20 samples with the spectral intensities closest to the target IM value is selected, in such a way that no scaling operations [48] are required. It is noteworthy that number of simulation levels has been defined based on a preliminary study, performed by considering different choices for the discretization of the IM range and for the 
numbers of ground motion samples to be considered at each IM interval. Based on this study, it was concluded that considering the hybrid reliability analysis method with 20 stripes and 20 samples conditional to the central IM interval values, corresponding to a total of 400 nonlinear structural analyses, allows to obtain accurate results at a fraction of the computational cost of the general, non-conditional, SS, requiring 1800 nonlinear structural analyses. Further details about the efficiency of the proposed hybrid approach compared to Subset Simulation are given in Appendix to this paper.

The demand hazard curves of the EDPs of interest are illustrated in Fig. 9a-d (with red solid lines for linear dampers and blue dashed lines for nonlinear dampers). For each curve, the reference performance point corresponding to the design condition, represented by the coordinates $\left\{d^{*}, v_{0}\left(d^{*}\right)\right\}$, is illustrated with a marker (a red circle for linear dampers and a blue square for nonlinear ones). The values of the coordinates of these points are also reported in Table 3. The values $v_{0}\left(d^{*}\right)$ are required to define the objective function of the RBO problems, since they represent the reference values (corresponding to dampers with nominal properties) with respect to which the maximum increment of the risk is evaluated. It is worth to observe that the reference MAF values estimated via probabilistic approach (i.e., MSA) are always higher than the design ones obtained through the deterministic design approach, as also observed in [35].

By comparing the demand hazard curves of the various EDPs, some common trends can be found. For instance, the damper velocity hazard curves (shown in Fig. 9f for sake of clarity) follow the same trends of the interstory drift (Fig. 9a) and damper stroke (Fig. 9d). According to these curves, for a given probability of exceedance, the response is higher in the case of nonlinear dampers, in particular within the range of MAFs lower than the design value. Conversely, the damper forces (Fig. 9e) exhibit opposite trends with respect to the velocities, and this is due to the fact that the nonlinear damper force cannot increase too much because of the velocity exponent $\alpha<1$.

Moreover, it is also worth to observe that the amplification rules provided in ASCE/SEI 41-13 [12] lead to different MAF values of damper force (Fig. 9e) and strokes (Fig. 9d) in linear and nonlinear viscous devices. In particular, the design force is more likely to be exceeded by the nonlinear dampers, with $v_{0}=3.16 \times 10^{-4} \mathrm{yrs}^{-1}$, than by the linear ones, with $v_{0}=1.37 \times 10^{-4} \mathrm{yrs}^{-1}$; the same trend is observed for the stroke, with $v_{0}=2.42 \times 10^{-4} \mathrm{yrs}^{-1}$ for the linear dampers and $v_{0}=4.04 \times 10^{-4} \mathrm{yrs}^{-1}$ for the nonlinear ones. These results are in agreement with the outcomes of previous studies [2,35], and confirm the need of improving the simplified approach provided by the codes, which does not ensure the same reliability levels for dampers with different nonlinear behaviour (i.e., different exponent $\alpha$ ).

Table 3. EDP design values $d^{*}$ and corresponding mean annual frequency (MAF) of exceedance $v_{0}$, for both linear $\left(\alpha_{0}=1.0\right)$ and nonlinear $\left(\alpha_{0}=0.3\right)$ dampers.

\begin{tabular}{llllll}
\hline EDPs & $\alpha_{0}=1.00$ & & \multicolumn{2}{l}{$\alpha_{0}=0.30$} \\
\cline { 2 - 3 } \cline { 5 - 6 } & $d^{*}$ & $v_{0}\left(\mathrm{x} 10^{-3}\right)\left[\mathrm{yrs}^{-1}\right]$ & & $d^{*}$ & $v_{0}\left(\mathrm{x} 10^{-3}\right)\left[\mathrm{yrs}^{-1}\right]$ \\
\hline$I D R$ & 0.0098 & 1.511 & 0.0098 & 1.623 \\
$\Delta_{d l}$ & $0.0509 \mathrm{~m}$ & 0.242 & $0.0426 \mathrm{~m}$ & 0.404 \\
$F_{d l}$ & $3614.20 \mathrm{kN}$ & 0.137 & $1811.82 \mathrm{kN}$ & 0.316 \\
$V_{b}$ & $7988.56 \mathrm{kN}$ & 1.619 & & $7615.73 \mathrm{kN}$ & 1.612 \\
$A$ & $4.65 \mathrm{~m} / \mathrm{s}^{2}$ & 1.929 & $5.49 \mathrm{~m} / \mathrm{s}^{2}$ & 1.723 \\
\hline
\end{tabular}

a)

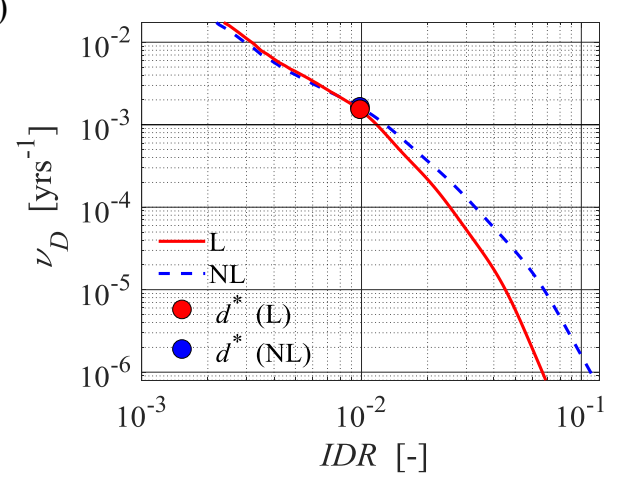

b)

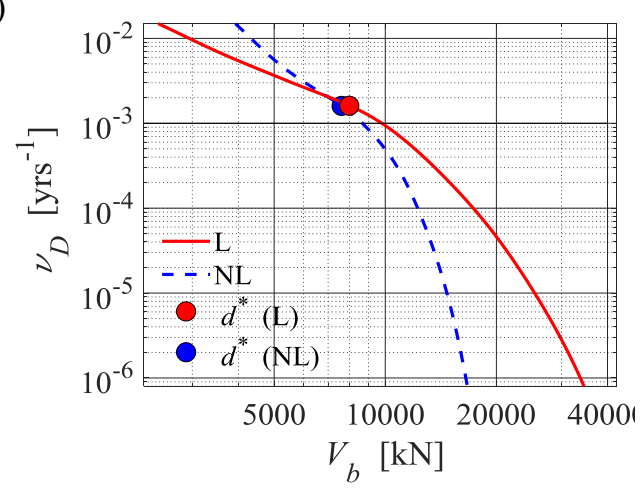


c)

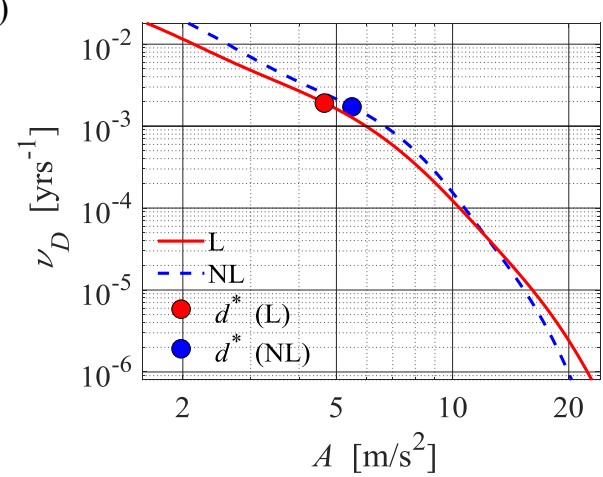

e)

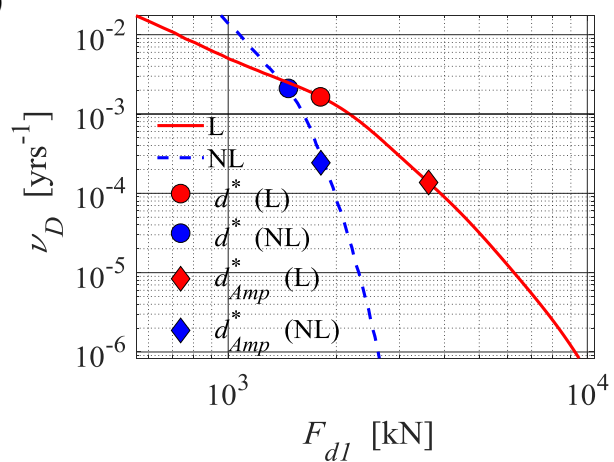

d)

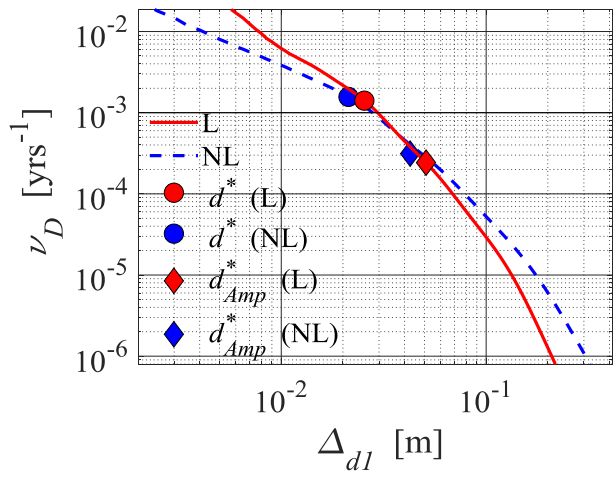

f)

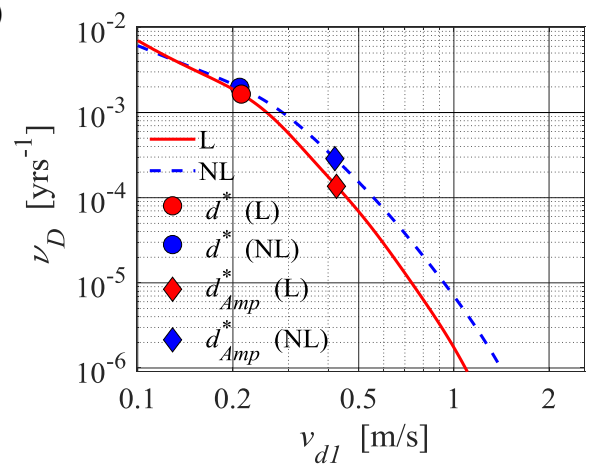

Fig. 9. Demand hazard curves for both linear $\left(\alpha_{0}=1.0\right)$ and nonlinear $\left(\alpha_{0}=0.3\right)$ dampers, and for the EDPs: a) maximum $I D R$; b) frame baseshear $\left.V_{b} ; \mathrm{c}\right)$ absolute acceleration $A$; d) force on damper $1 F_{d l}$; e) stroke on damper $1 \Delta_{d l}$; f) velocity of damper $1 v_{d l}$. Reference values (both design and amplified) are also shown with markers.

\section{RBO problem n. 1: effect of variable viscous coefficients (c)}

\subsection{Solution of RBO problem $n .1$ at the design condition}

This section addresses RBO problem 1, in which the vector of design variables $\hat{\boldsymbol{c}}=\left[\hat{c}_{1}, \hat{c}_{2}, \hat{c}_{3}\right]^{\mathrm{T}}$ collects the viscous coefficients of the dampers at the various storeys. These variables are assumed to vary independently, within the range $[-0.85,1.15] c_{0 i}$, where $c_{0 \mathrm{i}}$ is the nominal value of the viscous coefficient for the damper at the $i$-th storey.

The constrained RBO problem, as formulated in Eq. (7), has been solved five times, in order to separately find and discuss the worst consequences produced by the parameter variation on the five monitored EDPs (Table 3). Fig. 10 shows the history of the optimization process for the maximum interstory drift of the building with linear dampers as driving performance parameter. Fig. 10a shows the evolution of the rate of variation of each viscous coefficients $\hat{c}_{i}=\left(c_{i}-c_{0 i}\right) / c_{0 i}$ during the iterations of the optimization procedure. The starting condition corresponds to zero values of $\hat{c}_{i}$, i.e., the dampers have the nominal design properties. The COBYLA algorithm [32] tests different combinations of the perturbed coefficients until convergence is reached (after 24 iterations in this case), corresponding to a stable and constraint-compliant solution. Fig. 10b illustrates the corresponding evolution of the objective function, representing the normalized variation of the MAF of exceedance at the reference performance level of the monitored EDP. The optimal solution consists of:

- the viscous coefficient perturbations $\left\{\hat{c}_{1}, \hat{c}_{2}, \hat{c}_{3}\right\}=\{-15.0 \%,-15.0 \%,-15.0 \%\}$;

- $\mathrm{a}+15.0 \%$ variation of the MAF of exceeding the target drift 0.0098 (design value).

a)

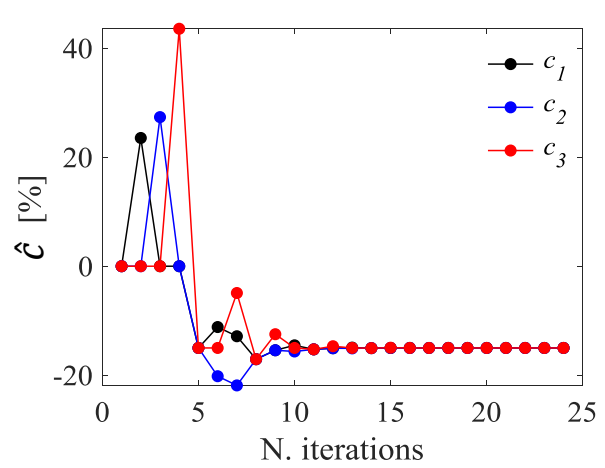

b)

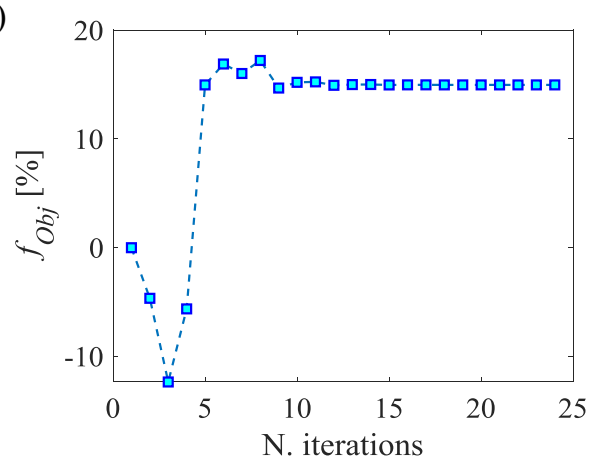


Fig. 10. Search for the optimal solution of the constrained RBO problem: a) dampers coefficients variation $\left(\hat{c}_{i}\right)$ and corresponding b) MAF of exceedance variation. EDP: IDR. Case with linear dampers $\left(\alpha_{0}=1.0\right)$.

The optimum solution provided by the RBO problem is compliant with the allowed tolerance on forces, i.e., the perturbed viscous coefficients lead to force values within the lower and upper bounds prescribed by the code.

The results obtained for all of the EDPs considered are summarized in Table 4, reporting, for each EDP and for both the linear and nonlinear dampers, the combinations of the most critical percentage variations of the damper viscous coefficients, and the corresponding percentage increment of the MAF (objective function). The negative variations of the design variables are highlighted in red, while the positive variations in black solid font.

Table 4. Results of the constrained RBO problem with variable viscous coefficients. The dampers coefficients variation $\left(\hat{c}_{i}\right)$ and corresponding MAF increment are reported for each EDP and for both the linear $L\left(\alpha_{0}=1.0\right)$ and nonlinear $N L\left(\alpha_{0}=0.3\right)$ dampers.

\begin{tabular}{|c|c|c|c|c|c|c|c|c|c|c|c|}
\hline & & \multicolumn{2}{|l|}{$I D R$} & \multicolumn{2}{|l|}{$\Delta_{d l}$} & \multicolumn{2}{|l|}{$F_{d 1}$} & \multicolumn{2}{|l|}{$V_{b}$} & \multicolumn{2}{|l|}{$A$} \\
\hline & & $\bar{L}$ & $\mathrm{NL}$ & $\mathrm{L}$ & $\mathrm{NL}$ & $\mathrm{L}$ & $\mathrm{NL}$ & $\mathrm{L}$ & $\mathrm{NL}$ & $\mathrm{L}$ & NL \\
\hline \multirow{3}{*}{$\begin{array}{l}\text { Viscous coefficient's } \\
\text { variations }\end{array}$} & $\hat{c}_{1}[\%]$ & -15.0 & -15.0 & -15.0 & -15.0 & +15.0 & +15.0 & +15.0 & +15.0 & +15.0 & +15.0 \\
\hline & $\hat{c}_{2}[\%]$ & -15.0 & -15.0 & -15.0 & -15.0 & +15.0 & -15.0 & +15.0 & +15.0 & -15.0 & -15.0 \\
\hline & $\hat{c}_{3}[\%]$ & -15.0 & -15.0 & +15.0 & +15.0 & +15.0 & +15.0 & +15.0 & +15.0 & -15.0 & -15.0 \\
\hline $\begin{array}{l}\text { Hazard demand } \\
\text { increment }\end{array}$ & $f_{O b j}[\%]$ & +15.0 & +12.0 & +20.0 & +16.0 & +59.0 & +271.7 & +12.0 & +22.7 & +6.0 & +5.4 \\
\hline
\end{tabular}

Based on the results reported in Table 4, the following observations can be made regarding the amount and sign of the viscous coefficients variations responsible for the highest increase of the MAF of exceedance of the monitored EDPs:

- The sets of varied viscous coefficients are found to be equal for the buildings with linear and nonlinear dampers for all of the EDPs, with the only exception of the damper force $\left(F_{d 1}\right)$, for which the maximum increments of the exceedance probability are obtained for different optimum solutions in terms of $\hat{c}_{i}$.

- In all the cases analysed, the viscous coefficients variations are always equal to the upper or to the lower bound of the constraints, and no intermediate admissible values of $\hat{c}_{i}$ are observed.

- The maximum MAF variations of the drift, the base-shear $\left(V_{b}\right)$ and the linear damper force $\left(F_{d l}\right)$ correspond to homogeneous viscous coefficients perturbations, i.e., with $\hat{c}>0$ on every damper.

- Differently, the maximum MAF variations of the remaining cases are produced by non-homogeneous viscous coefficients perturbations, i.e., having different signs on devices belonging to different floors.

For what concerns the effects induced by the viscous coefficients variations in terms of demand hazard variation, the following conclusions can be drawn:

- The seismic demand hazard sensitivity to viscous coefficients variations is generally low for the most of the EDPs, with percentage increments of the MAF of exceedance below the $22 \%$.

- Notably higher is the sensitivity shown by the damper force, with increments of $59.0 \%$ and $271.7 \%$ of the MAF of exceedance attained for, respectively, the linearly and nonlinearly damped building.

\subsection{Structural performance sensitivity at different MAF of exceedance}

The previous section focuses on the response variations at the design condition. However, the structural performance should be checked also at different conditions, corresponding to higher and lower values of the design MAF of exceedance. Thus, this section furnishes more useful insights into the effect of the damper uncertainties on the seismic performance, since it explores how the variations of the viscous coefficients obtained by solving the RBO problem 1 influence the response parameter variations for a wide range of MAF of exceedance values, spanning from the serviceability limit state condition to very rare conditions. For this purpose, MSA is repeatedly carried out by considering each time a set of the previously found worst combinations of $\hat{c}_{i}$ as per Table 4, for both the cases of linear and nonlinear dampers. This results in a total of 10 probabilistic analyses, which are carried out up to low values of the MAF of exceedance, of the order of $10^{-6} \mathrm{yrs}^{-1}$. It is noteworthy that this value is significantly lower than the one assumed for designing the seismic control system, which is around $10^{-3} \mathrm{yrs}^{-1}$ for all the EDPs except for the stroke and force of dampers, whose design MAF values vary between $10^{-3} \mathrm{yrs}^{-1}$ to $10^{-4} \mathrm{yrs}^{-1}$.

Fig. 11 shows the demand hazard curves of the base-shear (Fig. 11a) and of the maximum IDR (Fig. 11b) of the nonlinearly damped structure. The curve corresponding to dampers having nominal viscous coefficients (black solid curve) is compared to the curves related to the different worst combinations of varied viscous coefficients, represented by dashed lines of different colors. The black diamond marker in the figure represents the design condition considered for performing the RBO problem. 
Due to space constraints, the hazard curves of the other EDPs are not provided, but some results are reported for three MAF levels, namely $10^{-2}, 10^{-3}$, and $10^{-6}$, which are approximately representative of, respectively, the serviceability limit state, the ultimate limit state, and the failure conditions.

At each of these MAF levels, the percentage variation of the generic seismic demand parameter $D$ is computed through the following equation,

$$
\Delta d_{\max }\left(v_{D}\right)=100 \frac{d_{v a r}\left(v_{D}\right)-d_{0}\left(v_{D}\right)}{d_{0}\left(v_{D}\right)}
$$

in which $d_{v a r}$ represents the generic EDP value corresponding to the varied design variables, and $d_{0}$ is the EDP value corresponding to the nominal dampers condition. The resulting values are reported in Table 5-Table 7. Each row of the tables reports the variations of the EDP due to the dampers variations resulting from the solution of the 5 different RBO problems, each of which was solved to maximize the MAF of a specific EDP (different rows in the tables). Thus, the diagonal cells of the tables report the variations of the $i$-th EDP due to the dampers variations resulting from the RBO problem solved for the same EDP; the other terms of the tables furnish the variations of the $j$-th EDP due to the dampers variations resulting from the RBO problem solved for a different $k$-th EDP, for $k \neq j$. The maximum demand increments of each EDP are highlighted by black solid fonts.

a)

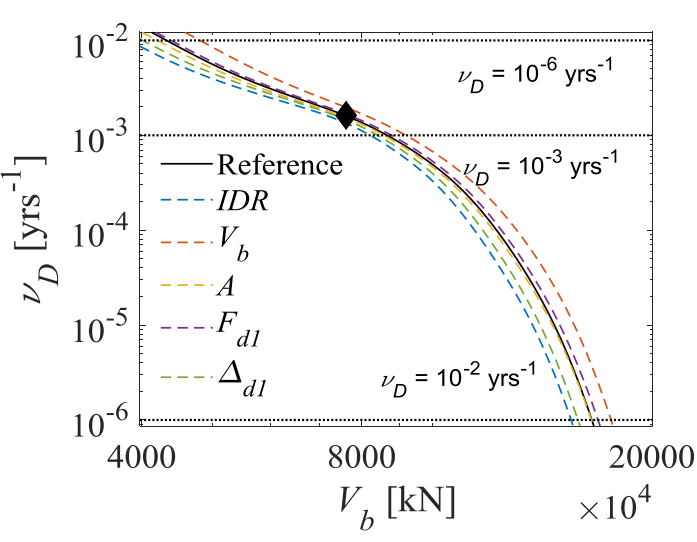

b)

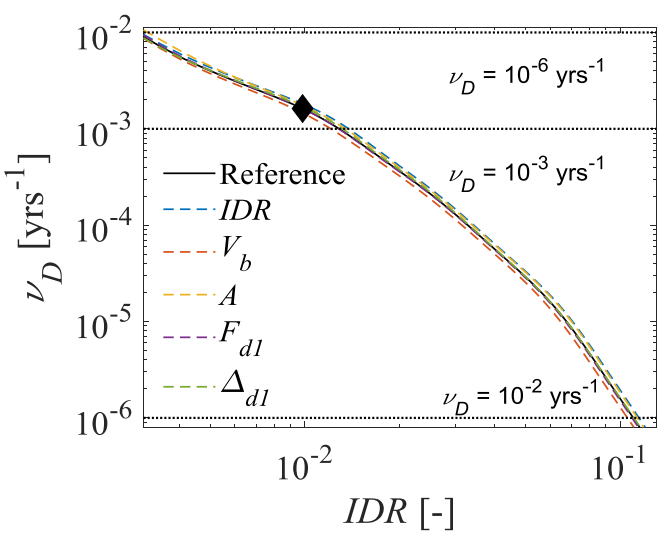

Fig. 11. Seismic demand variations for the EDPs a) $V_{b}$ and b) $I D R$ due to the combinations of varied viscous coefficients obtained by solving the 5 different RBO problems for the case with nonlinear dampers $\left(\alpha_{0}=0.3\right)$.

Table 5. Percentage variations of the performance demand due to the dampers properties variability at fixed MAF of exceedance: $v_{D}=10^{-2}$. Cases with linear (L) and nonlinear (NL) dampers.

\begin{tabular}{|c|c|c|c|c|c|c|c|c|c|c|}
\hline & \multicolumn{2}{|l|}{$I D R$} & \multicolumn{2}{|l|}{$V_{b}$} & \multicolumn{2}{|l|}{$A$} & \multicolumn{2}{|l|}{$F_{d l}$} & \multicolumn{2}{|l|}{$\Delta_{d l}$} \\
\hline & $\mathrm{L}$ & NL & $\mathrm{L}$ & NL & $\mathrm{L}$ & NL & $\mathrm{L}$ & NL & $\mathrm{L}$ & NL \\
\hline$I D R$ & +7.08 & +3.42 & -7.08 & -1.71 & +3.93 & +10.25 & -7.08 & +2.56 & +3.15 & -1.71 \\
\hline$V_{b}$ & -6.79 & -11.21 & +6.62 & +11.10 & -1.53 & -2.77 & +6.62 & +1.39 & -4.41 & -7.28 \\
\hline$A$ & +0.35 & -3.44 & +0.70 & +3.15 & +3.87 & +2.00 & +0.70 & +3.15 & -0.70 & -2.00 \\
\hline$F_{d l}$ & -11.03 & -12.96 & +9.66 & +12.04 & +9.66 & +5.56 & +9.66 & +8.80 & -11.72 & -11.11 \\
\hline$\Delta_{d l}$ & +7.05 & +13.25 & -7.69 & -13.25 & -3.85 & -22.89 & -7.69 & -18.07 & +6.41 & +22.89 \\
\hline
\end{tabular}

Table 6. Percentage variations of the performance demand due to the dampers properties variability at fixed MAF of exceedance: $v_{D}=10^{-3}$. Cases with linear (L) and nonlinear (NL) dampers.

\begin{tabular}{|c|c|c|c|c|c|c|c|c|c|c|}
\hline & \multicolumn{2}{|l|}{$I D R$} & \multicolumn{2}{|l|}{$V_{b}$} & \multicolumn{2}{|l|}{$A$} & \multicolumn{2}{|l|}{$F_{d l}$} & \multicolumn{2}{|l|}{$\Delta_{d l}$} \\
\hline & $\mathrm{L}$ & NL & $\mathrm{L}$ & NL & $\mathrm{L}$ & NL & $\mathrm{L}$ & $\mathrm{NL}$ & $\mathrm{L}$ & $\mathrm{NL}$ \\
\hline$I D R$ & +7.02 & +7.18 & -5.74 & -5.82 & +3.83 & +4.66 & -5.74 & -0.39 & +4.25 & +2.52 \\
\hline$V_{b}$ & -6.44 & -5.19 & +6.23 & +6.06 & -1.53 & -1.04 & +6.23 & +1.38 & -3.78 & -2.94 \\
\hline$A$ & -0.75 & +0.45 & +1.01 & -0.45 & +2.26 & +1.91 & +1.01 & +0.90 & -1.01 & -0.22 \\
\hline$F_{d l}$ & -11.24 & -14.20 & +10.09 & +13.89 & +10.09 & +13.89 & +10.09 & +13.89 & -11.01 & -13.89 \\
\hline$\Delta_{d l}$ & +7.11 & +9.16 & -6.26 & -8.94 & -3.89 & -5.37 & -6.26 & -5.72 & +7.44 & +9.66 \\
\hline
\end{tabular}

Table 7. Percentage variations of the performance demand due to the dampers properties variability at fixed MAF of exceedance: $v_{D}=10^{-6}$. Cases with linear (L) and nonlinear (NL) dampers. 


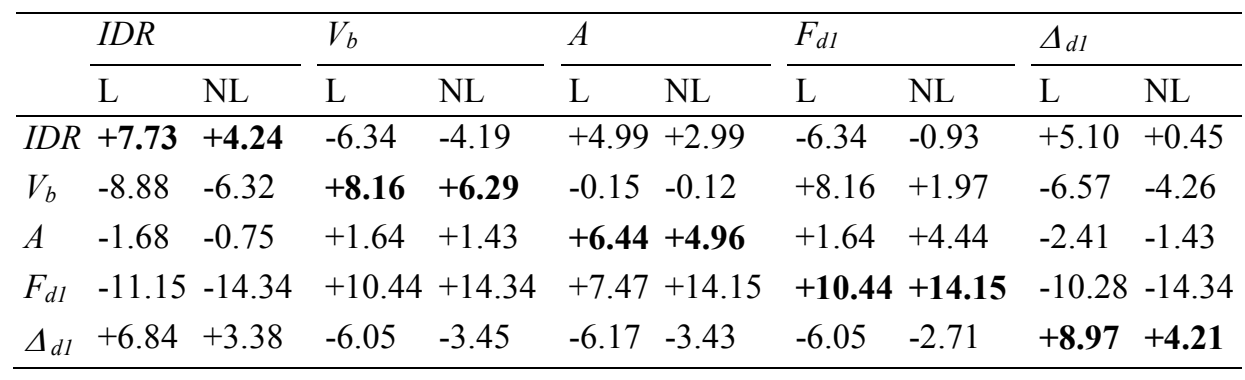

According to the results presented in the above tables, the following conclusions can be drawn:

- At the MAF level $v_{D}=10^{-3}$ (Table 6), approximately corresponding to the design hazard level at which the RBO is performed for almost all of the EDPs, the diagonal terms of Table 6 contain the maximum observed variations. Thus, the maximum demand variation of an EDP is generally due to damper coefficients perturbations resulting from the RBO problem which maximizes the MAF of that specific EDP.

- Because of a non-uniform effect of the damper coefficient perturbations over the whole range of MAFs, it is possible that, in correspondence of MAF levels other than $10^{-3}$, the demand increment is maximized by the set of varied viscous coefficients maximizing the probability of exceedance of other EDPs. This can be observed, for instance, for the interstory drift at $v_{D}=10^{-}$ ${ }^{2}$ (see Table 5 and also Fig. 11b), when nonlinear dampers are adopted. In this case, the maximum drift increment is observed for the perturbed viscous coefficients of the RBO problem performed on the maximum accelerations, although the present result is limited to this specific MAF level.

- The seismic demand increments due to admissible (i.e., code-complying) combinations of varied viscous coefficients are always lower than $15 \%$ for all the monitored EDP, type of dampers, and hazard levels investigated, with the only exception of the nonlinear damper stroke, whose amplification reaches the value of $23 \%$ at $v_{D}=10^{-2}$.

- Beyond the damper stroke in the nonlinear damper case, the most sensitive EDP is represented by the damper force, whose variations are generally higher in the building with nonlinear devices.

- Clear patterns and trends cannot be identified for the demand variations at the different hazard levels.

It is worth to highlight that the percentage variations reported in Table 5-Table 7 do not necessarily represent the maximum variations which one could expect due to the considered variability of the dampers properties. In fact, these variations have been obtained by using the worst combination of perturbed viscous parameters evaluated at the design condition (see Eqn. (3)). Higher variations may be observed by considering a different MAF of exceedance in the objective function of Eqn. (3). Nevertheless, the results shown above, given for the sake of completeness, provide some useful insight on the sensitivity of the seismic risk to variable dampers properties at MAF values lower than the design one.

Moreover, the variations reported in the tables above are consistent with the outcomes provided in Dall'Asta et al. (2017) [2] for the simpler case of a linear SDOF system with period of $1.0 \mathrm{~s}$. The same paper provides further details and explanations of the differences between the responses with linear and nonlinear dampers.

\section{RBO problem n. 2: effect of variable viscous parameters $(c, \alpha)$}

\subsection{Solution of RBO problem $n .2$ at the design condition}

Section 5 deals with the effect on the seismic performance of dampers with variable viscous coefficients. Despite the results are not entirely trivial, the most critical variations of the parameters are found to lie at the boundary of the admissible domain of variation. This section investigates a more complex problem, denoted as RBO problem n. 2, in which both the damper constitutive parameters are allowed to vary, while satisfying the tolerance criterion on the maximum damper force variations. The solution to this problem would not be possible without employing the optimization approach of Eqn. 8.

The design variables corresponding to the viscous parameters perturbations are collected in the vectors $\widehat{\boldsymbol{c}}=\left[\hat{c}_{1}, \hat{c}_{2}, \hat{c}_{3}\right]^{\mathrm{T}}$ and $\widehat{\boldsymbol{\alpha}}=\left[\hat{\alpha}_{1}\right.$, $\left.\hat{\alpha}_{2}, \hat{\alpha}_{3}\right]^{\mathrm{T}}$, and they are assumed to vary independently among the dampers at the different storeys, while respecting the tolerance bounds of Eq. (6). This RBO problem, mathematically formulated in Eq. (9), is solved for each of the five monitored EDPs of Table 3. Fig. 12 illustrates the evolution of the design parameter variations during the optimization process carried out by assuming as EDP the force of the linear damper at the first-storey $\left(F_{d l}\right)$. Fig. 13a shows, for the same EDP, the evolution of the objective function, i.e., the normalized MAF variation, which attains the optimal value of $+63.0 \%$ in correspondence of the following set of design variables:

- variations of the viscous coefficient, $\left\{\hat{c}_{1}, \hat{c}_{2}, \hat{c}_{3}\right\}=\{+33.9 \%,+15.0 \%,+15.0 \%\}$;

- variations of the velocity exponents, $\left\{\hat{\alpha}_{1}, \hat{\alpha}_{2}, \hat{\alpha}_{3}\right\}=\{+11.6 \%, 0.0 \%, 0.0 \%\}$. 
It is worth to recall that the aim of the present study is to evaluate how effective the code tolerance criteria can be in controlling the performance parameter variations, without explicitly taking into consideration whether the parameter perturbations resulting from the RBO problem solution can actually be physically attained through the manufacturing process. In light of this, it is noteworthy that although the normalized variation of the viscous constant of the damper at the first storey is quite high, it is indeed compatible with the tolerance constraint, and thus it complies with current seismic codes. However, further experimental tests would be required to shed light on the variation of the dampers constitutive parameters related to the manufacturing process, an information not available to date.
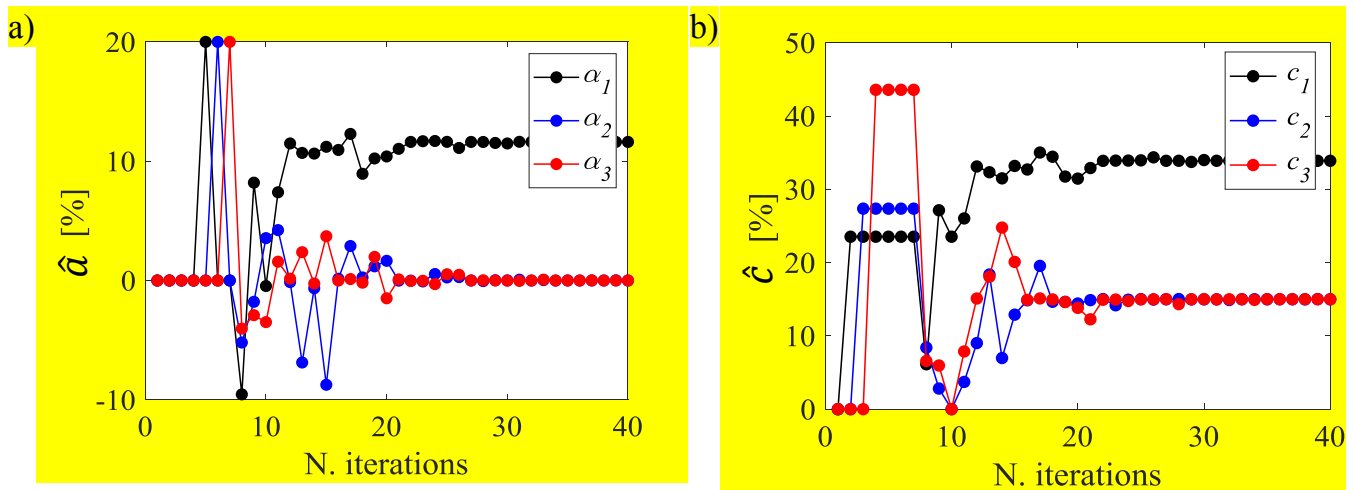

Fig. 12. Search for the optimal solution of the constrained RBO problem: a) velocity exponents variation $\left(\hat{\alpha}_{i}\right)$, and b) dampers coefficients variation $\left(\hat{c}_{i}\right)$. EDP: force on damper $1 F_{d l}$. Case with linear dampers $\left(\alpha_{0}=1.0\right)$.

a)

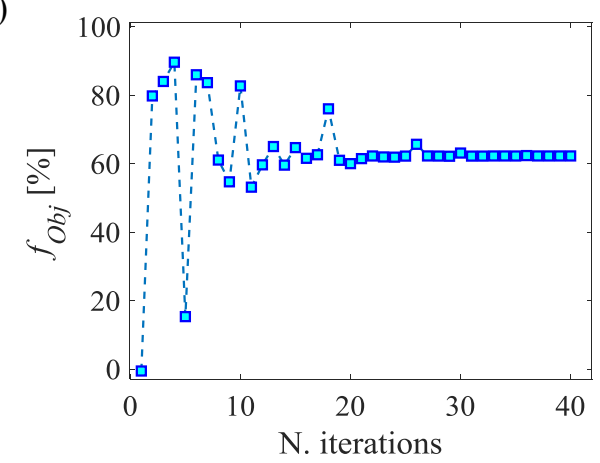

b)

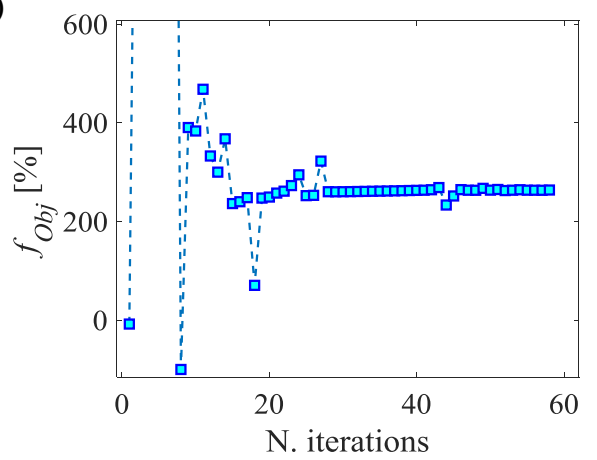

Fig. 13. Objective function-iterations for the constrained RBO problem: MAF of exceedance variation. EDP: force on damper $1 F_{d I}$. Case with: a) linear dampers $\left(\alpha_{0}=1.0\right)$ and $\left.b\right)$ nonlinear dampers $\left(\alpha_{0}=0.3\right)$.

As shown in Fig. 14, the above variations are following the constraints imposed in terms of damper forces. It is worth to observe that, due to the variability on both $c_{1}$ and $\alpha_{1}$, the damper $D_{1}$ (Fig. 14a) exhibits a force value equal to the upper bound of the tolerance range at the design velocity $v^{*}=0.27 \mathrm{~m} / \mathrm{s}$, while for $v>v^{*}$, the force attains values larger than 1.15 times the nominal value, as allowed by the acceptance criteria currently provided by seismic codes [12].

Fig. 13b illustrates the evolution of the objective function for the optimization processes carried out by considering, as EDP, the force of the nonlinear damper with $\alpha_{0}=0.3$ at the first-storey $\left(F_{d 1}\right)$. The optimal value of $+271.7 \%$ attained by the normalized MAF variation is due to the following set of design variables:

- variations of the viscous coefficient, $\left\{\hat{c}_{1}, \hat{c}_{2}, \hat{c}_{3}\right\}=\{+15.0 \%,-15.0 \%,+15.0 \%\}$;

- variations of the velocity exponents, $\left\{\hat{\alpha}_{1}, \hat{\alpha}_{2}, \hat{\alpha}_{3}\right\}=\{+0.0 \%, 0.0 \%, 0.0 \%\}$.

It can be observed that the worst combination of the perturbed viscous properties for this case corresponding to nonlinear dampers coincides with the solution found by the previous RBO problem $\mathrm{n}$. 1, and consists of variations of only the viscous coefficients.

The results obtained for the RBO problems performed on all of the considered EDPs are summarized in Table 8, reporting, for each EDP and for both the linear and nonlinear dampers, the combinations of the most critical percentage variations of the viscous constitutive parameters, and the corresponding percentage increment of the MAF of exceedance. The negative variations of the design variables are highlighted in red, while the positive variations in black solid font. 
a)

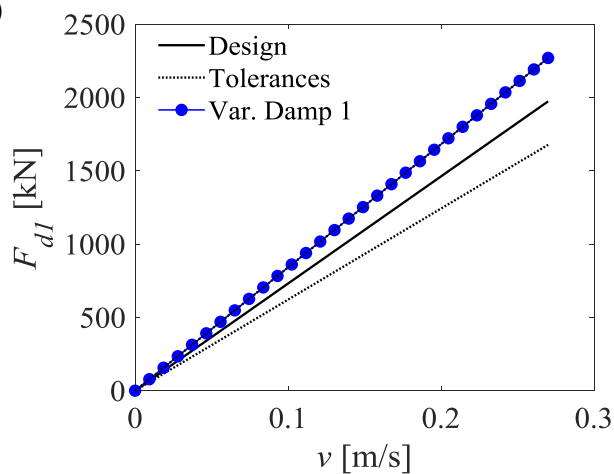

b)

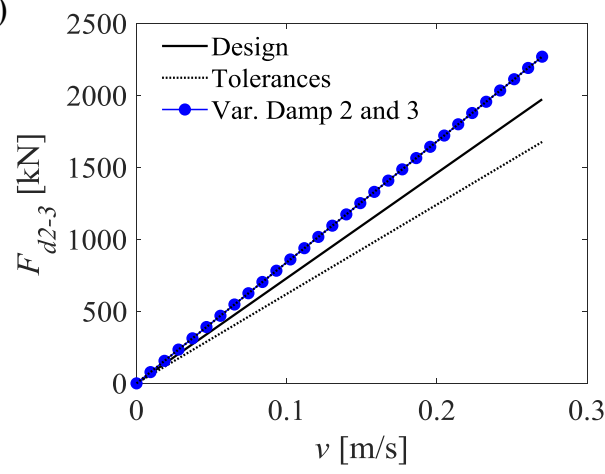

Fig. 14. Compliance with the constraint imposed on damper force of the worst viscous parameters combination found by solving the RBO problem on the EDP $F_{d l}$. Nonlinear damper response for the a) device floor 1 and b) device floor 2 and 3.

Table 8. Results of the constrained RBO problem with variable viscous parameters. The dampers constitutive parameters variation $\left(\hat{\alpha}_{i}, \hat{c}_{i}\right)$ and corresponding MAF increment are reported for each EDP and for both the linear $L\left(\alpha_{0}=1.0\right)$ and nonlinear $N L\left(\alpha_{0}=0.3\right)$ dampers.

\begin{tabular}{|c|c|c|c|c|c|c|c|c|c|c|c|}
\hline & & $I D R$ & & $\Delta_{d l}$ & & $F_{d 1}$ & & $V_{b}$ & & $A$ & \\
\hline & & $\mathrm{L}$ & $\mathrm{NL}$ & $\mathrm{L}$ & $\mathrm{NL}$ & $\mathrm{L}$ & $\mathrm{NL}$ & $\mathrm{L}$ & $\mathrm{NL}$ & $\mathrm{L}$ & NL \\
\hline \multirow{3}{*}{$\begin{array}{l}\text { Viscous coefficient's } \\
\text { variation }\end{array}$} & $\hat{c}_{1}[\%]$ & -15.0 & -15.0 & -15.0 & -15.0 & +33.9 & +15.0 & +15.0 & +15.0 & +15.0 & +15.0 \\
\hline & $\hat{c}_{2}[\%]$ & -15.0 & -15.0 & -15.0 & -22.3 & +15.0 & -15.0 & +15.0 & +15.0 & -15.0 & -15.0 \\
\hline & $\hat{c}_{3}[\%]$ & -15.0 & -15.0 & +33.9 & +15.0 & +15.0 & +15.0 & +15.0 & +15.0 & -27.0 & -15.0 \\
\hline \multirow{3}{*}{$\begin{array}{l}\text { Exponent } \alpha \\
\text { variation }\end{array}$} & $\hat{\alpha}_{1}[\%]$ & 0.0 & 0.0 & 0.0 & 0.0 & +11.6 & 0.0 & 0.0 & 0.0 & 0.0 & 0.0 \\
\hline & $\hat{\alpha}_{2}[\%]$ & 0.0 & 0.0 & 0.0 & -24.9 & 0.0 & 0.0 & 0.0 & 0.0 & 0.0 & 0.0 \\
\hline & $\hat{\alpha}_{3}[\%]$ & 0.0 & 0.0 & +11.6 & 0.0 & 0.0 & 0.0 & 0.0 & 0.0 & -11.2 & 0.0 \\
\hline $\begin{array}{l}\text { Hazard demand } \\
\text { increment }\end{array}$ & $f_{O b j}[\%]$ & +15.0 & +12.0 & +24.0 & +19.0 & +63.0 & +271.7 & +12.0 & +22.7 & +7.7 & +5.4 \\
\hline
\end{tabular}

It is worth to note that the results of RBO problem n. 2 are less predictable than those of RBO problem 1, and do not lie at the boundary of the admissible domain, thus demonstrating the usefulness of the proposed optimization approach for the sensitivity problem solution.

Based on the results reported in Table 8, the following observations can be made on the influence of variable viscous parameters:

- In four cases out of 10 , the worst combinations of viscous parameters involve the velocity exponents variability $(\hat{\alpha} \neq 0)$. These cases are: the EDPs $F_{d l}$ and $A$ for the buildings with linear dampers, and the EDP $\Delta_{d l}$ for both the linear and nonlinear damped systems. In the remaining six cases, the RBO problem 2 yields the same results as the RBO problem 1, revealing a reduced sensitivity of the monitored objective functions to the velocity exponent variability.

- The increments of the MAF of exceedance observed in the four cases characterized by combined variations of $\hat{c}$ and $\hat{\alpha}$ are slightly higher (of maximum four percentage points) than the ones corresponding to perturbations of the viscous coefficients only (see Table 4 for the results of RBO problem 1).

- The maximum admissible positive variation observed on the viscous coefficients is equal to $+33.9 \%$, corresponding to a velocity exponent variation of $+11.6 \%$. The maximum admissible negative variation observed on the viscous coefficients is equal to $+27.0 \%$, corresponding to a velocity exponent variation of $-11.2 \%$.

- $\quad$ The higher sensitivity of the damper force demand parameter $\left(F_{d l}\right)$ with respect to the DVs, already observed in RBO problem 1 , is confirmed.

Summarizing the results obtained thus far, it is possible to state that the variability of the velocity exponent $(\alpha)$, usually disregarded by seismic codes [12,13], may affect the seismic hazard of some EDPs, although in this specific case study the effects are reduced.

\subsection{Structural performance sensitivity at different MAF of exceedance}

This section investigates the influence of the sets of varied viscous parameters of Table 8 on the seismic demand at different MAF levels. As done for RBO Problem 1, the demand variations are evaluated for the following MAF levels: $10^{-2}, 10^{-3}$, and $10^{-6}$, and the results are collected in Table 9-Table 11. 
Table 9. Percentage variations of the performance demand due to the dampers properties variability at fixed MAF of exceedance: $v_{D}=10^{-2}$. Cases with linear (L) and nonlinear (NL) dampers.

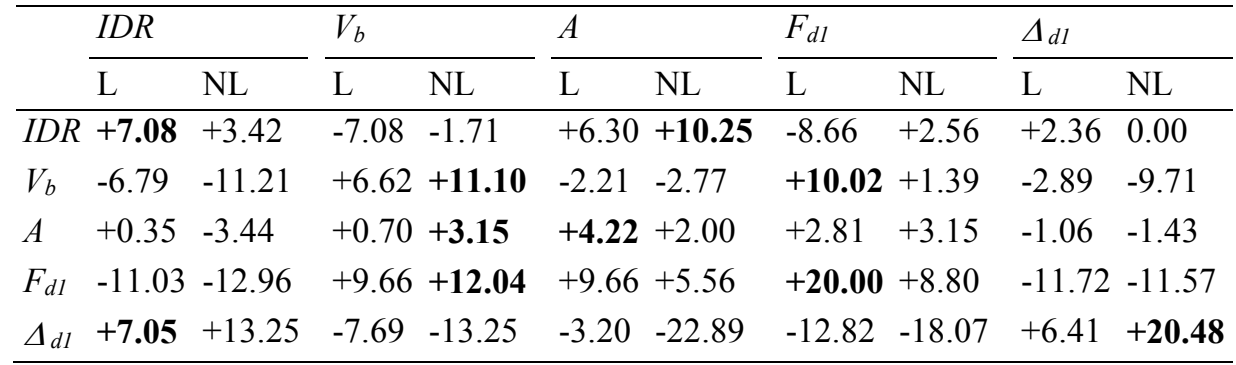

Table 10. Percentage variations of the performance demand due to the dampers properties variability at fixed MAF of exceedance: $v_{D}=10^{-3}$. Cases with linear (L) and nonlinear (NL) dampers.

\begin{tabular}{|c|c|c|c|c|c|c|c|c|c|c|}
\hline & \multicolumn{2}{|l|}{$I D R$} & \multicolumn{2}{|l|}{$V_{b}$} & \multicolumn{2}{|l|}{$A$} & \multicolumn{2}{|l|}{$F_{d l}$} & \multicolumn{2}{|l|}{$\Delta_{d l}$} \\
\hline & $\mathrm{L}$ & NL & $\mathrm{L}$ & NL & $\mathrm{L}$ & $\mathrm{NL}$ & $\mathrm{L}$ & NL & $\mathrm{L}$ & NL \\
\hline$I D R$ & +7.02 & +7.18 & -5.74 & -5.82 & +5.53 & +4.66 & -7.23 & -0.39 & +3.40 & +4.08 \\
\hline$V_{b}$ & -6.44 & -5.19 & +9.23 & +6.06 & -2.60 & -1.04 & +9.09 & +1.38 & -2.15 & -3.98 \\
\hline$A$ & -0.75 & +0.45 & +1.01 & -0.45 & +2.51 & +1.91 & +2.27 & +0.90 & -1.13 & 0.00 \\
\hline$F_{d l}$ & -11.24 & -14.20 & +10.09 & +13.89 & +10.32 & +13.89 & +21.79 & +13.89 & -10.78 & -13.89 \\
\hline$\Delta_{d l}$ & +7.11 & +9.66 & -6.26 & -8.94 & -3.89 & -5.37 & -12.18 & -5.72 & +7.78 & +10.02 \\
\hline
\end{tabular}

Table 11. Percentage variations of the performance demand due to the dampers properties variability at fixed MAF of exceedance: $v_{D}=10^{-6}$. Cases with linear (L) and nonlinear (NL) dampers.

\begin{tabular}{|c|c|c|c|c|c|c|c|c|c|c|}
\hline & \multicolumn{2}{|l|}{$I D R$} & \multicolumn{2}{|l|}{$V_{b}$} & \multicolumn{2}{|l|}{$A$} & \multicolumn{2}{|l|}{$F_{d l}$} & \multicolumn{2}{|l|}{$\Delta_{d l}$} \\
\hline & $\mathrm{L}$ & NL & $\mathrm{L}$ & NL & $\mathrm{L}$ & NL & $\mathrm{L}$ & NL & $\mathrm{L}$ & NL \\
\hline$I D R$ & +7.73 & +4.24 & -6.34 & -4.19 & +8.51 & +2.99 & -7.95 & -0.93 & +4.01 & +1.25 \\
\hline$V_{b}$ & -8.88 & -6.32 & +8.16 & +6.29 & -1.21 & -0.12 & +13.06 & +1.97 & -5.33 & -5.35 \\
\hline$A$ & -1.68 & -0.75 & +1.64 & +1.43 & +6.87 & +4.96 & +6.10 & +4.44 & -2.68 & -0.56 \\
\hline$F_{d l}$ & -11.15 & -14.34 & +10.44 & +14.34 & +7.09 & +14.15 & +20.51 & +14.15 & -9.74 & -14.34 \\
\hline$\Delta_{d l}$ & +6.84 & +3.38 & -6.05 & -3.45 & -7.01 & -3.43 & -13.11 & -2.71 & +10.19 & +4.39 \\
\hline
\end{tabular}

The following conclusions can be drawn regarding the effects on the seismic demand of dampers with varied constitutive parameters:

- The increments of seismic demand due to combined variations of viscous coefficients and velocity exponents are generally higher than those due to variation of the viscous coefficients only (RBO problem 1).

- The most sensitive EDP is the damper force, as also observed in RBO problem 1.

- Unlike the case with variable viscous coefficients, in this case the added variability of $\alpha$ is responsible for a further increment of the force demand at the MAF $v_{D}=10^{-3}$ and $v_{D}=10^{-6}$, where the percentage variations increase from nearly $11 \%$ in the case of fixed $\alpha$ to around $21 \%$ in the case of variable $\alpha$.

- Force reliability factor observed in this application is larger than factors currently proposed by codes [12,13], equal to 1.15 , and the results suggest that a deeper investigation of the topics is necessary.

- Clear patterns and trends cannot be identified for the demand variations at the different hazard levels.

As for the case of Table 5-Table 7, these variations have been observed by considering the same optimal damper variations for all the three MAF levels.

\section{Conclusions}

In this paper, an innovative approach is proposed for investigating the seismic risk sensitivity of structures with respect to uncertain system parameters having a bounded range of variation. This method is of interest for structures equipped with anti-seismic devices, such as isolators or dampers. It consists in solving a reliability-based optimization (RBO) problem that allows to identify the worst combination of a set of input parameters that maximize a probabilistic objective function, expressing the variation of the mean annual frequency of exceedance of the performance parameters of interest.

The capability of the proposed approach for evaluating the potentially worst conditions that jeopardize the system performance is analysed by considering a specific application, investigating the influence of the variability of the constitutive parameters (due to 
the uncertainties in the devices manufacturing process) of linear and nonlinear fluid viscous dampers on the seismic risk of a lowrise steel building.

Based on the results of the application of the RBO problem, the following conclusions are drawn for the specific problem analysed.

- The seismic performance of the building may drop as a result of the effects of variability of the damper properties, with different levels of increase of the demand hazard depending on the type of dampers (linear or nonlinear) and the specific EDP monitored.

- The various response parameters considered are differently affected by the damper property variability, and the most sensitive response quantity is represented by the damper force, whose design value may experience increments of the corresponding MAF of exceedance spanning from $63 \%$ to $272 \%$ for, respectively, linear and nonlinear dampers, because of admissible combined variations of the viscous parameters $c$ and $\alpha$.

- The effect of the variation of the damper constitutive parameters is found to be limited for most of the monitored EDPs. However, among all the EDPs analysed, the damper forces have shown to be very sensitive to the damping constitutive parameters variation, in particular when the velocity exponents $\alpha$ deviate from their nominal values.

- The employed RBO techniques has been shown to be robust in dealing with several design variables with independent constrained domains of variation.

Despite the developed application has a demonstrative character, it serves to show that the proposed methodology provides outcomes which would not be achieved without an optimization-based approach. In fact, the proposed approach permits to directly establish a relationship between the parameters controlling the design process and the global seismic risk. In particular, it allows to verify the reliability factors proposed by seismic codes for safety checks, and eventually to better calibrate their values to properly account for the tolerances allowed for supplemental damping devices. For example, the results concerning the considered case study, show that the reliability factors for the damper force according to ASCE/SEI 41-13 may need to be increased, with respect to the values currently provided, to consider the influence of the variability of the velocity exponent $\alpha$. Obviously, a more precise proposal about this point requires a larger set of experimental and numerical investigations, also aimed at providing more insight into the likely range of variation of the damper properties.

Moreover, the proposed method can be employed not only to further investigate the problem of viscous dampers, but also to analyze the effect of the uncertainties of other types of seismic devices, such as hysteretic dampers or isolation bearings.

\section{Acknowledgements}

The study reported in this paper was sponsored by the Italian Department of Civil Protection within the Reluis-DPC Projects 20142018. The authors gratefully acknowledge this financial support.

\section{References}

[1] Tubaldi E, Freddi F, Barbato M. Probabilistic seismic demand model for pounding risk assessment. Earthq Eng Struct Dyn 2016;45:1743-58. doi:10.1002/eqe.2725.

[2] Dall'Asta A, Scozzese F, Ragni L, Tubaldi E. Effect of the damper property variability on the seismic reliability of linear systems equipped with viscous dampers. Bull Earthq Eng 2017;15:5025-53. doi:10.1007/s10518-017-0169-8.

[3] Tubaldi E, Barbato M, Dall'Asta A. Influence of Model Parameter Uncertainty on Seismic Transverse Response and Vulnerability of Steel-Concrete Composite Bridges with Dual Load Path. J Struct Eng 2012;138:363-74. doi:10.1061/(ASCE)ST.1943-541X.0000456.

[4] Porter KA, Beck JL, Shaikhutdinov R V. Sensitivity of building loss estimates to major uncertain variables. Earthq Spectra 2002;18:719-43. doi:10.1193/1.1516201.

[5] Lee TH, Mosalam KM. Seismic demand sensitivity of reinforced concrete shear-wall building using FOSM method. Earthq Eng Struct Dyn 2005;34:1719-36. doi:10.1002/eqe.506.

[6] Kwon OS, Elnashai AS. The effect of material and ground motion uncertainity on the seismic vulnerability of RC strcutures. Eng Struct 2006;28:289-303.

[7] Dolsek M. Incremental dynamic analysis with consideration of modeling uncertainties. Earthq Eng Struct Dyn 2009;38:805-25. doi:10.1002/eqe.869.

[8] Celik OC, Ellingwood BR. Seismic fragilities for non-ductile reinforced concrete frames - Role of aleatoric and epistemic uncertainties. Struct Saf 2010;32:1-12. doi:10.1016/j.strusafe.2009.04.003.

[9] Ibarra LF, Krawinkler H. Global Collapse of Frame Structures under Seismic Excitations. Evaluation 2005:1-301. doi:10.1002/eqe.

[10] Thomson A, Whittaker AS, Fenves GL, Mahin SA. Property Modification Factors for Elastomeric Seismic Isolation Bearings. 12Wcee2000 1999:1-8. 
[11] ASCE. ASCE/SEI 7-10: Minimum design loads for buildings and other structures. 2010. doi:10.1061/9780784412916.

[12] ASCE 41-13: Seismic Evaluation and Retrofit Rehabilitation of Existing Buildings 2014. doi:10.1061/9780784412855.

[13] European Committee for Standardization. EN 15129:2010 - Antiseismic devices. 2010.

[14] Constantinou M, Tsopelas P, Kasalanati A, Wolff E. Property modification factors for seismic isolation bearings 1999.

[15] Micozzi F, Ragni L, Dall'Asta A. Statistical modelling of HDNR bearing properties variability for the seismic response of isolated structures. 6th Eur. Conf. Comput. Mech. (ECCM 6). 11 - 15 June 2018, Glas. UK, 2018.

[16] Constantinou MC, Whittaker AS, Kalpakidis Y, Fenz DM, Warn GP. Performance of Seismic Isolation Hardware under Service and Seismic Loading - Technical Report MCEER-07-0012. Mceer 2007:471.

[17] Kumar M, Whittaker AS, Constantinou MC. Seismic isolation of nuclear power plants using elastomeric bearings. 2015.

[18] Der Kiureghian A, Liu P. Structural Reliability under Incomplete Probability Information. J Eng Mech 1986;112:85-104. doi:10.1061/(ASCE)0733-9399(1986)112:1(85).

[19] De Angelis M, Patelli E, Beer M. Advanced Line Sampling for efficient robust reliability analysis. Struct Saf 2015;52:170-82. doi:10.1016/j.strusafe.2014.10.002.

[20] Saltelli A, Ratto M, Andres T, Campolongo F, Cariboni J. Global sensitivity analysis: the primer 2008.

[21] Patelli E, Pradlwarter HJ, Schuller GI. Global sensitivity of structural variability by random sampling. Comput Phys Commun 2010.

[22] Barbato M, Gu Q, Conte JP. Probabilistic Push-Over Analysis of Structural and Soil-Structure Systems. J Struct Eng 2010;136:1330-41. doi:10.1061/(ASCE)ST.1943-541X.0000231.

[23] Barbato M, Zona A, Conte JP. Probabilistic nonlinear response analysis of steel-concrete composite beams. J Struct Eng 2014;140. doi:10.1061/(ASCE)ST.1943-541X.0000803.

[24] Lupoi G, Franchin P, Lupoi A, Pinto PE. Seismic Fragility Analysis of Structural Systems. J Eng Mech 2006;132:385-95. doi:10.1061/(ASCE)0733-9399(2006)132:4(385).

[25] Au S. Reliability-based design sensitivity by efficient simulation. Comput Struct 2005;83:1048-61. doi:10.1016/j.compstruc.2004.11.015.

[26] Au SK, Beck JL. Subset Simulation and its Application to Seismic Risk Based on Dynamic Analysis. J Eng Mech 2003;129:901-17. doi:10.1061/(ASCE)0733-9399(2003)129:8(901).

[27] Dall'Asta A, Ragni L, Scozzese F, Tubaldi E. Reliability of systems equipped with viscous dampers with variable properties. ECCOMAS Congr 2016 - Proc 7th Eur Congr Comput Methods Appl Sci Eng 2016;3. doi:10.7712/100016.2201.7987.

[28] Zona A, Ragni L, Dall'Asta A. Sensitivity-based study of the influence of brace over-strength distributions on the seismic response of steel frames with BRBs. Eng Struct 2012;37:179-92.

[29] Jensen HA, Sepulveda JG. On the reliability-based design of structures including passive energy dissipation systems. Struct Saf 2011;34:390-400. doi:10.1016/j.strusafe.2011.09.005.

[30] Jensen HA, Valdebenito MA, Schuëller GI, Kusanovic DS. Reliability-based optimization of stochastic systems using line search. Comput Methods Appl Mech Engrg 2009;198:3915-24.

[31] Taflanidis A, Beck J. Life-cycle cost optimal design of passive dissipative devices. Struct Saf 2009;31:508-22.

[32] Powell MJD. A view of algorithms for optimization without derivatives. Math Today-Bulletin Inst Math Its Appl 2007;43:170-4.

[33] Altieri D, Tubaldi E, De Angelis M, Patelli E, Dall'Asta A. Reliability-based optimal design of nonlinear viscous dampers for the seismic protection of structural systems. Bull Earthq Eng 2018;16:963-82. doi:10.1007/s10518-017-0233-4.

[34] Baker J, Cornell C. Vector-valued ground motion intensity measures for probabilistic seismic demand analysis. Berkeley: 2006.

[35] Dall'asta A, Tubaldi E, Ragni L. Influence of the nonlinear behavior of viscous dampers on the seismic demand hazard of building frames. Earthq Eng Struct Dyn 2016;45:149-69. doi:10.1002/eqe.2623.

[36] Ohtori Y, Christenson RE, Spencer BF, Dyke SJ. Benchmark Control Problems for Seismically Excited Nonlinear Buildings. J Eng Mech 2004;130:366-85. doi:10.1061/(ASCE)0733-9399(2004)130:4(366).

[37] Barroso LR, Winterstein S. Probabilistic seismic demand analysis of controlled steel moment-resisting frame structures. Earthq Eng Struct Dyn 2002;31:2049-66. doi:10.1002/eqe.201.

[38] Gupta A, Krawinkler H. Seismic Demands for Performance Evaluation of Steel Moment Resisting Frame Structures. Dr Diss Stanford Univ 1999:1-379. 
[39] Lavan O, Avishur M. Seismic behavior of viscously damped yielding frames under structural and damping uncertainties. Bull Earthq Eng 2013;11:2309-32. doi:10.1007/s10518-013-9479-7.

[40] Jcss. Probabilistic Model Code - Part 1. Struct Saf 2001:65.

[41] Institution BS. Eurocode 0 - Basis of structural design. En 2002;3:89. doi:10.1680/cien.144.6.8.40609.

[42] Bradley BA. A comparison of intensity-based demand distributions and the seismic demand hazard for seismic performance assessment. Earthq Eng Struct Dyn 2013;42:2235-53. doi:10.1002/eqe.2322.

[43] Jayaram N, Baker JW. Efficient sampling and data reduction techniques for probabilistic seismic lifeline risk assessment. Earthq Eng Struct Dyn 2010;39:1109-31. doi:10.1002/eqe.988.

[44] Rubinstein R, Kroese D. Simulation and the Monte Carlo method 2016.

[45] Aslani H, Miranda E. Probability-based seismic response analysis. Eng Struct 2005;27:1151-63. doi:10.1016/j.engstruct.2005.02.015.

[46] Bradley BA, Dhakal RP, MacRae GA, Cubrinovski M. Prediction of spatially distributed seismic demands in specific structures: Structural response to loss estimation. Earthq Eng Struct Dyn 2010;39:591-613. doi:10.1002/eqe.955.

[47] Katsanos EI, Sextos AG, Manolis GD. Selection of earthquake ground motion records: A state-of-the-art review from a structural engineering perspective. Soil Dyn Earthq Eng 2010;30:157-69. doi:10.1016/j.soildyn.2009.10.005.

[48] Bradley BA, Burks LS, Baker JW. Ground motion selection for simulation-based seismic hazard and structural reliability assessment. Earthq Eng Struct Dyn 2015;44:2321-40. doi:10.1002/eqe.2588.

[49] Patelli E. COSSAN: A multidisciplinary software suite for uncertainty quantification and risk management. Handb Uncertain Quantif 2017:1909-77. doi:10.1007/978-3-319-12385-1_59.

[50] Lavan O, Dargush GF. Multi-objective evolutionary seismic design with passive energy dissipation systems. J Earthq Eng 2009;13:758-90. doi:10.1080/13632460802598545.

[51] Pavlou E, Constantinou MC. Response of Nonstructural Components in Structures with Damping Systems. J Struct Eng 2006;132:1108-17. doi:10.1061/(ASCE)0733-9445(2006)132:7(1108).

[52] Constantinou M, Symans M. Experimental and Analytical Investigation of Seismic Response of Structures with Supplemental Fluid Viscous Dampers 1992:NCEER-92-0032.

[53] Castellano MG, Borella R, Infanti S. Experimental characterization of nonlinear fluid viscous dampers according to the New European Standard. 5th Eur Conf Struct Control 2012:1-8.

[54] Symans MD, Constantinou MC. Passive Fluid Viscous Damping Systems for Seismic Energy Dissipation. J Earthq Technol 1998;35:185-206.

[55] Pollini N, Lavan O, Amir O. Towards realistic minimum-cost optimization of viscous fluid dampers for seismic retrofitting. Bull Earthq Eng 2016;14:971-98. doi:10.1007/s10518-015-9844-9.

[56] Tubaldi E, Ragni L, Dall'Asta A. Probabilistic seismic response assessment of linear systems equipped with nonlinear viscous dampers. Earthq Eng Struct Dyn 2015;44:101-20. doi:10.1002/eqe.2461.

[57] Mazzoni S, Mazzoni S, Mckenna F, Scott MH, Fenves GL. The Open System for Earthquake Engineering Simulation (OpenSEES) User Command-Language Manual 2006.

[58] Yu YJ, Tsai KC, Li CH, Weng YT, Tsai CY. Earthquake response analyses of a full-scale five-story steel frame equipped with two types of dampers. Earthq Eng Struct Dyn 2013;42:1301-20. doi:10.1002/eqe.2273.

[59] Scozzese F, Terracciano G, Zona A, Della Corte G, Dall'Asta A, Landolfo R. Analysis of seismic non-structural damage in single-storey industrial steel buildings. Soil Dyn Earthq Eng 2018;114:505-19. doi:10.1016/j.soildyn.2018.07.047.

[60] Seo CY, Karavasilis TL, Ricles JM, Sause R. Seismic performance and probabilistic collapse resistance assessment of steel moment resisting frames with fluid viscous dampers. Earthq Eng Struct Dyn 2014;43:2135-54. doi:10.1002/eqe.2440.

[61] Atkinson GM, Silva W. Stochastic modeling of California ground motions. Bull Seismol Soc Am 2000;90:255-74. doi:10.1785/0119990064.

[62] Boore DM. Simulation of Ground Motion Using the Stochastic Method. Seism. Motion, Lithospheric Struct. Earthq. Volcan. Sources Keiiti Aki Vol., Basel: Birkhäuser Basel; 2003, p. 635-76. doi:10.1007/978-3-0348-8010-7_10.

[63] Jalayer F, Beck JL. Effects of two alternative representations of ground-motion uncertainty on probabilistic seismic demand assessment of structures. Earthq Eng Struct Dyn 2008;37:61-79. doi:10.1002/eqe.745.

[64] FEMA-350: Recommended seismic design criteria for new steel moment-frame buildings. Washington DC: 2000.

[65] Hwang JS, Lin WC, Wu NJ. Comparison of distribution methods for viscous damping coefficients to buildings. Struct 
Infrastruct Eng 2013;9:28-41. doi:10.1080/15732479.2010.513713.

[66] Whittle JK, Williams MS, Karavasilis TL, Blakeborough A. A comparison of viscous damper placement methods for improving seismic building design. J Earthq Eng 2012;16:540-60. doi:10.1080/13632469.2011.653864.

[67] Pekcan G, Mander J, Chen S. Design and Retrofit Methodology for Building Structures with Supplemental Energy Dissipating Systems. 1999.

[68] Tubaldi E, Barbato M, Dall'Asta A. Efficient approach for the reliability-based design of linear damping devices for seismic protection of buildings. ASCE-ASME J Risk Uncertain Eng Syst Part A Civ Eng 2016;2. doi:10.1061/AJRUA6.0000858.

[69] Au S, Wang Y. Engineering risk assessment with subset simulation 2014.

\section{List of Acronyms}

COBYLA Constrained Optimization BY Linear Approximation

DV Design Variable

EDP Engineering Demand Parameter

FOSM First-Order Second-Moment

IDR Interstory-Drift Ratio

IM Intensity Measure

L Linear damper

MAF Mean Annual Frequency

MSA Multiple-Stripe Analysis

NL Nonlinear damper

RBO Reliability-Based Optimization

SDOF Single Degree Of Freedom

\section{Appendix. Hybrid probabilistic approach validation}

This Appendix provides a quantitative validation, in terms of efficiency and accuracy, of the hybrid probabilistic approach used in this paper within the RBO algorithm. To this aim, the demand hazard curves stemming from the hybrid method with multiple-stripe analysis (MSA) are compared to those deriving from a direct probabilistic approach, the latter consisting on Subset Simulation.

More precisely, the hazard curves derived by averaging 20 independent simulation runs of Subset Simulation are assumed as reference solution. Each Subset Simulation run is performed using 300 samples per level and 6 simulation levels with a progressive exceedance probability of $p_{0}=10 \%$; the EDP maximum interstory drift $(I D R)$ is assumed as driving variable, while the other EDPs (maximum base-shear, maximum damper force and stroke) are assumed as auxiliary variables (Au and Wang, 2014 [69]).

Fig. A. 1a-e compares the demand hazard curves for different EDPs obtained via MSA and via Subset Simulation (average of 20 runs), for the building equipped with linear dampers. Results concerning the case with nonlinear dampers are presented in Fig. A. 2a-e.

The reasonably good match between the curves from Subset Simulation (black solid lines) and the curves from MSA (red dashed lines) offers a qualitative proof of the accuracy of the method. Moreover, Table A. 1 reports the percentage errors of the estimates of MSA with respect to Subset Simulation for both the cases with linear and nonlinear dampers. These errors are evaluated in terms of EDP values, $d\left(v_{D}\right)$, corresponding to different levels of the mean annual frequency of exceedance $v_{D}: 10^{-3}, 10^{-4}, 10^{-5}, 10^{-6}, 10^{-7}$. Denoting with $d_{S S}\left(v_{D}\right)$ the EDP value estimated via Subset Simulation at the mean annual rate $v_{D}$, and with $d_{M S A}\left(v_{D}\right)$ the same quantity estimated via MSA, the error $e_{\%}$ associated with the MSA estimate is evaluated as follows.

$$
e_{\%}\left(v_{D}\right)=100 \frac{d_{M S A}\left(v_{D}\right)-d_{S S}\left(v_{D}\right)}{d_{S S}\left(v_{D}\right)}
$$

Table A. 1. EDPs percentage errors stemming from multiple-stripe analysis with respect to subset simulation. Cases with linear and nonlinear viscous dampers are compared.

\begin{tabular}{|c|c|c|c|c|c|c|c|c|c|c|}
\hline & \multicolumn{2}{|c|}{$v_{D}=10^{-3}$} & \multicolumn{2}{|c|}{$v_{D}=10^{-4}$} & \multicolumn{2}{|c|}{$v_{D}=10^{-5}$} & \multicolumn{2}{|c|}{$v_{D}=10^{-6}$} & \multicolumn{2}{|c|}{$v_{D}=10^{-7}$} \\
\hline & $\mathrm{L}$ & $\mathrm{NL}$ & $\mathrm{L}$ & NL & $\mathrm{L}$ & NL & $\mathrm{L}$ & NL & $\mathrm{L}$ & NL \\
\hline$I D R$ & 3.8 & -7.6 & 5.0 & 13.3 & 1.3 & 8.8 & 0.3 & 3.1 & 0.9 & 5.0 \\
\hline $\mathrm{Vb}$ & 1.1 & -0.4 & 3.0 & 5.8 & 0.5 & 1.3 & 0.6 & -0.2 & -4.1 & -2.9 \\
\hline $\mathrm{F}_{\mathrm{d} 1}$ & 5.6 & 1.8 & 5.4 & 4.2 & 4.6 & 2.0 & -7.7 & -3.0 & -6.2 & -3.8 \\
\hline$\Delta_{\mathrm{d} 1}$ & -16.9 & -0.8 & 9.2 & 22.1 & -1.3 & 7.2 & 2.0 & 0.4 & -1.2 & -9.3 \\
\hline
\end{tabular}


The highest errors, although negligible, are equal to $-16.9 \%$ and $22.1 \%$, and they are observed for the stroke demand of, respectively, the linear and nonlinear damper cases, as highlighted by bold font in Table A. 1. Except for these two values, in all the other cases very low percentage errors are observed. Based on these results, MSA can efficiently replace Subset Simulation for the solution of the RBO problem, without affecting the accuracy of the estimated probabilities.

The main advantage of the proposed hybrid approach over Subset Simulation is its notably lower computational cost. In fact, the time required to perform a single run of Subset Simulation (with 6 simulation levels, $\mathrm{p}_{0}=10 \%$ and 300 samples each) is about 6 times higher than that required by the hybrid approach (with a total amount of 400 analyses). These computations have been carried out on a Desktop Computer with Intel Xeon processor E5-2630V3, 8 cores (16 threads), and 32 GB of RAM.

a)

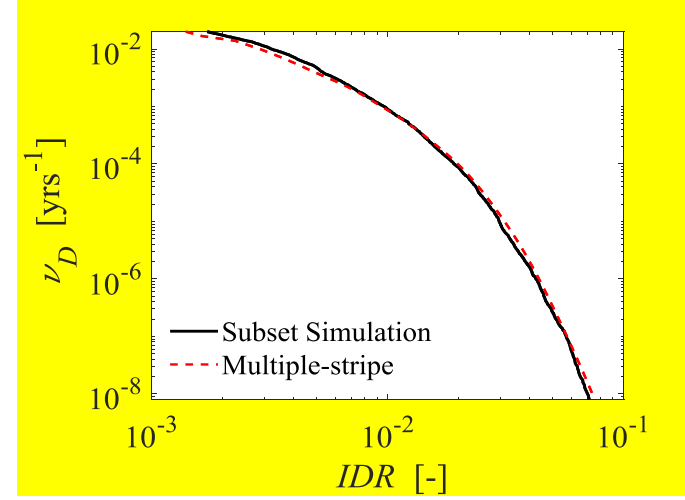

c)

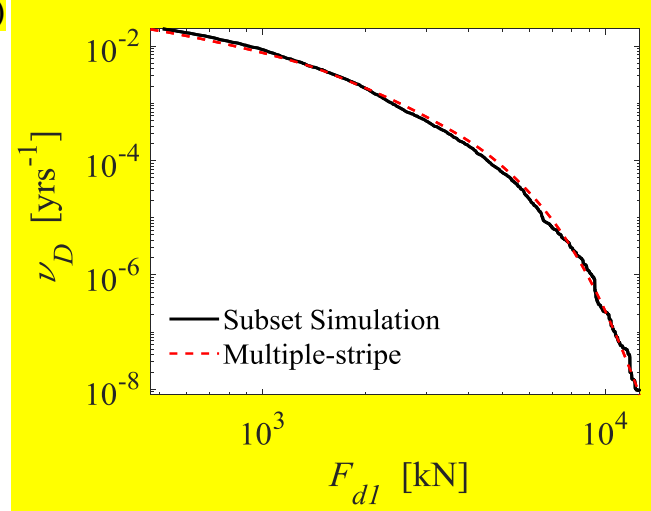

b)

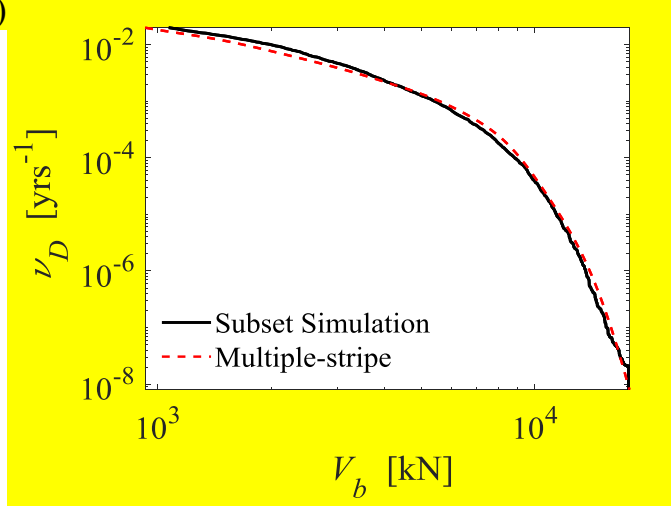

d)

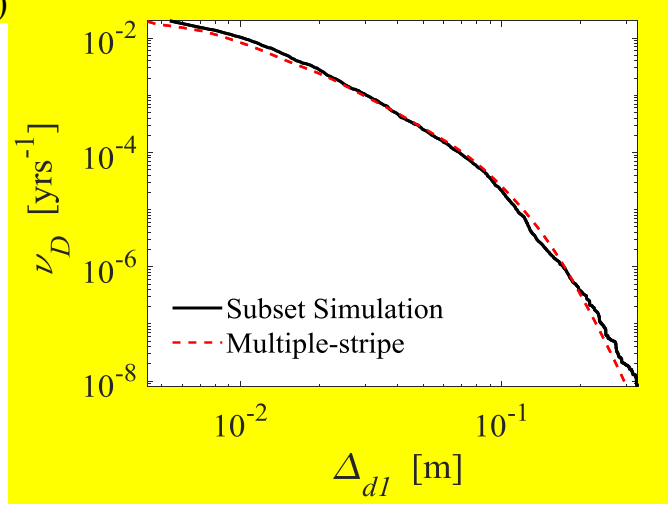

Fig. A. 1. Demand hazard curves from multiple-stripe analysis (MSA) vs subset simulation. Case with linear viscous dampers

a)

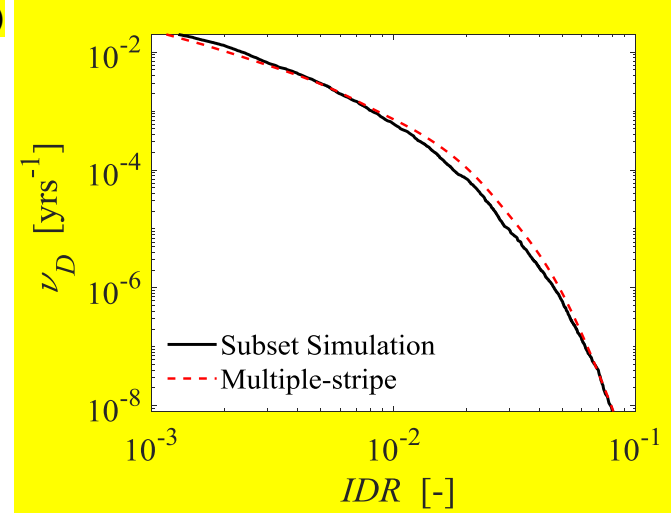

c)

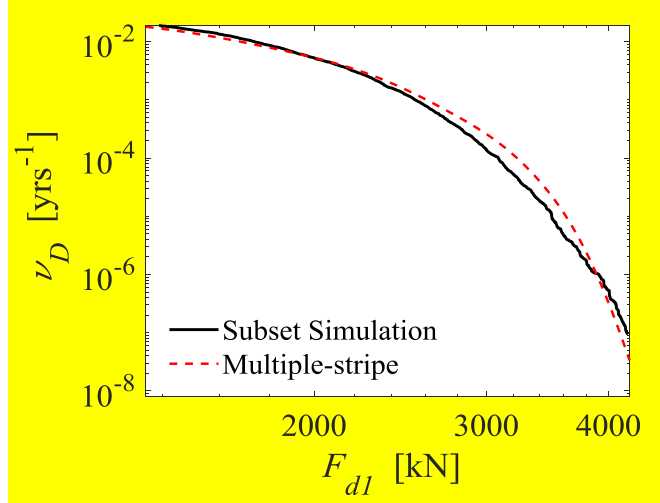

b)

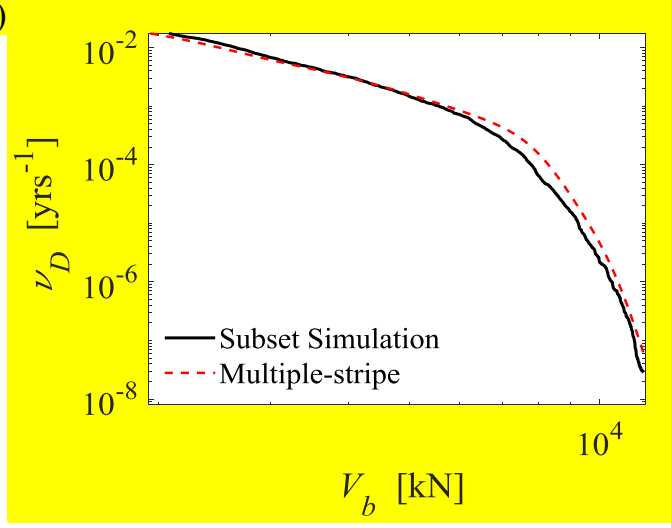

d)

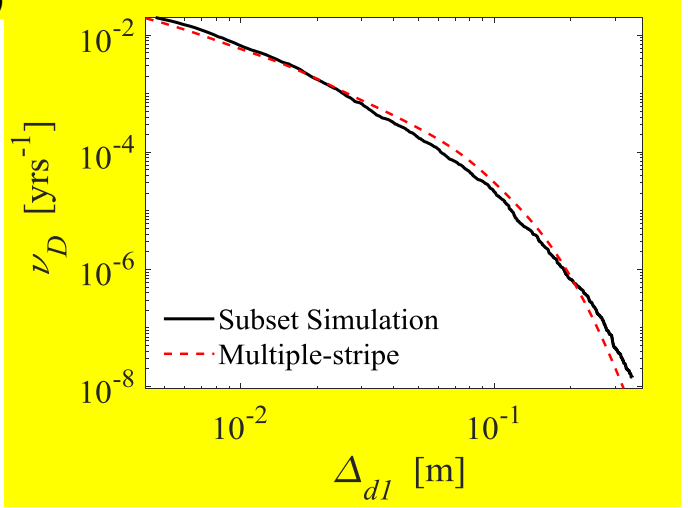


Fig. A. 2. Demand hazard curves from multiple-stripe analysis (MSA) vs subset simulation. Case with nonlinear viscous dampers 